
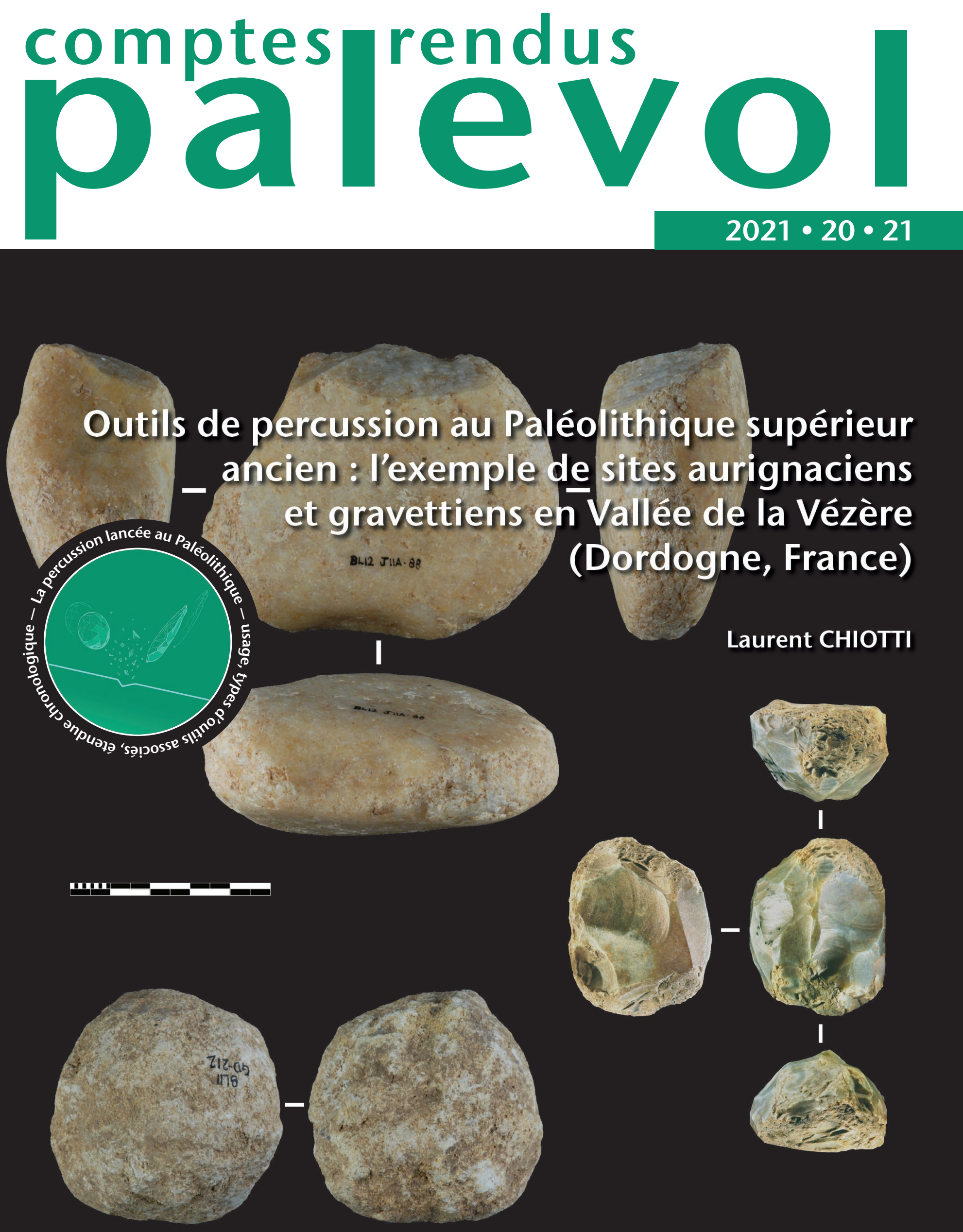
DiRECTEURS DE LA PUBLICATION / PUBLICATION DIRECTORS:

Bruno David, Président du Muséum national d'Histoire naturelle

Étienne Ghys, Secrétaire perpétuel de l'Académie des sciences

RÉDACTEURS EN CHEF / EDITORS-IN-CHIEF: Michel Laurin (CNRS), Philippe Taquet (Académie des sciences)

AssistANTE DE RÉDACTION / AssistANT EDITOR: Adenise Lopes (Académie des sciences; cr-palevol@academie-sciences.fr)

MISE EN PAGE / PAGE LAYOUt: Audrina Neveu (Muséum national d'Histoire naturelle; audrina.neveu@mnhn.fr)

RÉVISIONS LINGUISTIQUES DES TEXTES ANGLAIS / ENGLISH LANGUAGE REVISIONS: Kevin Padian (University of California at Berkeley)

RÉDACTEURS ASSOCIÉS / ASSOCIATE EDITORS ( ${ }^{*}$, took charge of the editorial process of the article/a pris en charge le suivi éditorial de l'article):

Micropaléontologie/Micropalaeontology

Maria Rose Petrizzo (Università di Milano, Milano)

Paléobotanique/Palaeobotany

Cyrille Prestianni (Royal Belgian Institute of Natural Sciences, Brussels)

Métazoaires/Metazoa

Annalisa Ferretti (Università di Modena e Reggio Emilia, Modena)

Paléoichthyologie/Palaeoichthyology

Philippe Janvier (Muséum national d'Histoire naturelle, Académie des sciences, Paris)

Amniotes du Mésozoïque/Mesozoic amniotes

Hans-Dieter Sues (Smithsonian National Museum of Natural History, Washington)

Tortues/Turtles

Juliana Sterli (CONICET, Museo Paleontológico Egidio Feruglio, Trelew)

Lépidosauromorphes/Lepidosauromorphs

Hussam Zaher (Universidade de São Paulo)

Oiseaux/Birds

Eric Buffetaut (CNRS, École Normale Supérieure, Paris)

Paléomammalogie (mammifères de moyenne et grande taille)/Palaeomammalogy (large and mid-sized mammals)

Lorenzo Rook (Università degli Studi di Firenze, Firenze)

Paléomammalogie (petits mammifères sauf Euarchontoglires)/Palaeomammalogy (small mammals except for Euarchontoglires)

Robert Asher (Cambridge University, Cambridge)

Paléomammalogie (Euarchontoglires)/Palaeomammalogy (Euarchontoglires)

K. Christopher Beard (University of Kansas, Lawrence)

Paléoanthropologie/Palaeoanthropology

Roberto Macchiarelli (Université de Poitiers, Poitiers)

Archéologie préhistorique/Prehistoric archaeology

Marcel Otte* (Université de Liège, Liège)

RÉFÉRÉS / REVIEWERS: https://sciencepress.mnhn.fr/fr/periodiques/comptes-rendus-palevol/referes-du-journal

COUVERTURE / COVER:

Made from the Figures of the article.

Comptes Rendus Palevol est indexé dans / Comptes Rendus Palevol is indexed by:

- Cambridge Scientific Abstracts

- Current Contents ${ }^{\circledR}$ Physical

- Chemical, and Earth Sciences ${ }^{\circledR}$

- ISI Alerting Services ${ }^{\circledR}$

- Geoabstracts, Geobase, Georef, Inspec, Pascal

- Science Citation Index ${ }^{\circledR}$, Science Citation Index Expanded ${ }^{\circledR}$

- Scopus ${ }^{\circledR}$.

Les articles ainsi que les nouveautés nomenclaturales publiés dans Comptes Rendus Palevol sont référencés par / Articles and nomenclatural novelties published in Comptes Rendus Palevol are registered on:

- ZooBank ${ }^{\circledR}$ (http://zoobank.org)

Comptes Rendus Palevol est une revue en flux continu publiée par les Publications scientifiques du Muséum, Paris et l'Académie des sciences, Paris Comptes Rendus Palevol is a fast track journal published by the Museum Science Press, Paris and the Académie des sciences, Paris

Les Publications scientifiques du Muséum publient aussi / The Museum Science Press also publish:

Adansonia, Geodiversitas, Zoosystema, Anthropozoologica, European Journal of Taxonomy, Naturae, Cryptogamie sous-sections Algologie, Bryologie, Mycologie.

L'Académie des sciences publie aussi / The Académie des sciences also publishes:

Comptes Rendus Mathématique, Comptes Rendus Physique, Comptes Rendus Mécanique, Comptes Rendus Chimie, Comptes Rendus Géoscience, Comptes Rendus Biologies.

Diffusion - Publications scientifiques Muséum national d'Histoire naturelle

CP 41 - 57 rue Cuvier F-75231 Paris cedex 05 (France)

Tél. : 33 (0)1 40794805 / Fax: 33 (0)1 40793840

diff.pub@mnhn.fr / https://sciencepress.mnhn.f

Académie des sciences, Institut de France, 23 quai de Conti, 75006 Paris.

(C) Publications scientifiques du Muséum national d'Histoire naturelle / @ Académie des sciences, Paris, 2021

ISSN (imprimé / print): 1631-0683/ ISSN (électronique / electronic): 1777-571X 


\title{
Outils de percussion au Paléolithique supérieur ancien : l'exemple de sites aurignaciens et gravettiens en Vallée de la Vézère (Dordogne, France)
}

\author{
Laurent CHIOTTI \\ Histoire Naturelle de l'Homme Préhistorique (HNHP), Muséum national d'Histoire naturelle, \\ CNRS, abri Pataud, 20 rue du Moyen-Âge, FR-24620 Les Eyzies-de-Tayac (France) \\ Ichiotti@mnhn.fr
}

Soumis le 25 mai 2020 | Accepté le 3 juin 2020 | Publié le 4 juin 2021

MOTS CLÉS

Percuteur,

percussion,

Aurignacien,

Gravettien.

Chiotti L. 2021. - Outils de percussion au Paléolithique supérieur ancien : l'exemple de sites aurignaciens et gravettiens en Vallée de la Vézère (Dordogne, France), in Viallet C., Bourguignon L., Lemorini C. \& Ortega† I. (eds), La percussion lancée au Paléolithique : identification de son usage, types d'outils associés et étendue chronologique. Comptes Rendus Palevol 20 (21) : 373-400. https://doi.org/10.5852/cr-palevol2021v20a21

\section{RÉSUMÉ}

Le travail présenté ici est issu d'une étude plus globale de l'ensemble des matériaux lithiques hors silex (MLHS), menée sur plusieurs sites du Paléolithique supérieur ancien de la vallée de la Vézère (Dordogne, France). Parmi les très nombreux objets entrant dans cette catégorie, nous ne présenterons ici que les pièces montrant des traces d'utilisation en percussion. Bien que ces traces soient généralement présentes sur des galets, nous verrons que d'autres types de matériaux ont été utilisés, avec notamment le réemploi de silex initialement taillés pour d'autres usages. L'étude concerne trois sites aurignaciens (les abris Blanchard et Castanet à Sergeac et l'abri Cellier à Tursac) et les niveaux du Gravettien final de deux autres sites (les abris Pataud et de Laugerie-Haute aux Eyzies-de-Tayac). Les collections étudiées ont des origines très diverses : collections issues de fouilles anciennes, collecte dans les déblais de fouilles anciennes et fouilles actuelles. Une analyse macroscopique a révélé l'existence d'une certaine diversité fonctionnelle parmi ces outils utilisés en percussion.

\footnotetext{
ABSTRACT

Percussion tools from early Upper Palaeolithic: Example of Aurignacian and Gravettian sites from the Vézère Valley (Dordogne, France).

The work presented in this paper is the result of a more general study of all the non-flint lithic materials carried out on several sites of the early Upper Paleolithic of the Vézère valley (Dordogne, France). Among the many objects in this category, we present here only those showing traces of percussion use. Although these traces are most often present on river pebbles, other types of materials have also been used, including the re-use of flints originally knapped for something else. The study concerns three Aurignacian rock shelter sites (abri Blanchard and abri Castanet in Sergeac and Abri Cellier in Tursac) and the Final Gravettian levels of two other rock shelter sites (Pataud and Laugerie-Haute in Les Eyzies-de-Tayac). The collections studied have very different origins: collections from old excavations, collection from the spoil of old excavations and current excavations. Thus, these collections are very heterogeneous. Among the studied material, a macroscopic analysis revealed the presence of different categories of percussion tools, corresponding to diverse uses.
} 
Among the studied material, a macroscopic analysis revealed the presence of different categories of percussion tools, corresponding to diverse uses:

-82 hammerstones for flint knapping.

These are the most numerous. Among them we observe the greatest variability, both in terms of dimensions and raw materials. They are usually medium sized objects: between 50 and $100 \mathrm{~mm}$ for a weight of 90 to $500 \mathrm{~g}$. Most are made of quartz, but there is great variability in the choice of raw material including gneiss, granite and quartzite.

-43 pounding stones.

They are river pebbles that show much more violent traces of impacts on the extremities or edges, often with splinters, sometimes altering the shape and volume of the support. They are more numerous on the Aurignacian sites. They are always among the biggest river pebbles with traces of percussion: between 80 and $130 \mathrm{~mm}$ for a weight of 200 to $900 \mathrm{~g}$. A piece from Castanet measures $1400 \mathrm{~g}$. The raw materials used are almost exclusively quartz and quartzite.

- 22 choppers/hammerstones.

These are river pebbles with voluntary flake detachments to create more or less sharp ridges, which typologically can be called choppers. They all have very significant percussion and crush marks on their ridges. Most of them come from the Aurignacian sites. They are very similar in traces, dimensions and raw materials to pounding stones and probably had the same use.

-3 soft stone hammerstones.

These are two spherical calcareous pieces and a small flint pebble with a very thick and soft cortex. They are among the smallest hammerstones and were probably used for flint knapping. -20 "maillets" (mallets).

According to the definition of S. de Beaune (2000), these are small river pebbles, usually elongated, showing groups of more or less intense percussion marks, which can form a clear depression on the river pebble surface. They have relatively small dimensions: between 60 and $110 \mathrm{~mm}$ in length for 20 to $70 \mathrm{~mm}$ in width and weights from 20 to $130 \mathrm{~g}$. The raw materials used are varied, but are generally fine-grained and compact rocks (lithographic limestone, diorite, etc.). Quartz is never used. -49 flint objects reused in percussion.

Most of these objects are cores, but there is also one large flake, some carinate scrapers and one carinate scraper rought-out. Some have such intensive percussion marks that the edges have almost completely disappeared, which gives them an almost spherical appearance. With lengths between 30 and $80 \mathrm{~mm}$ for a weight of 30 to $300 \mathrm{~g}$, they are among the smallest hammerstones. It is in the Gravettian sites that they are most numerous. We have several hypotheses concerning their uses: as hammerstones for flint knapping, bush-hammers or even lighters.

-9 intermediate pieces.

They are all from Castanet. They are quite voluminous flints with traces of percussion and crushing on two opposing areas. In most cases, these traces are relatively less intense.

-25 anvils.

These are objects of very varying sizes, weighing from $300 \mathrm{~g}$ to several kilos. Many of them have traces of anvil use, but also traces of use as pounding stones, flint knapping hammerstones, cutting supports, grinders or for chopper shaping. Quartz is the dominant material, but almost all the quartz pieces were also used as hammerstones.

The five Aurignacian and Final Gravettian sites of the Vézère valley have all delivered quite large quantities of objects bearing use-stigmata of activities related to percussion, with a total of 275 pieces. In addition to being abundant, the percussion material in the five sites is relatively diverse. There are different categories of tools that revealed different uses, not just flint knapping. On the other hand, it appears that for each given type of tool, the uses seem to be the same in both cultures.

KEY WORDS

Percussion tool,

percussion,

Aurignacian,

Gravettian.
Among the raw materials used, quartz pebbles are widely used, irrespective of site and culture. Alongside this dominant material, a considerable diversity of materials was selected. This diversity may vary depending on the type of object/use, but also the site/culture

\section{INTRODUCTION}

Les niveaux du Paléolithique supérieur livrent très fréquemment des quantités importantes de matériaux lithiques hors silex (MLHS). Ces derniers comprennent essentiellement des galets de rivière de différentes natures, mais aussi des fossiles, des cristaux, etc. (Chiotti et al. 2018). Par le passé, ces objets n'ont que rarement fait l'objet d'analyses approfondies; dans nombre de fouilles anciennes, ils n'ont tout simplement pas été collectés.

Le travail présenté ici est issu d'une étude plus globale de l'ensemble des MLHS menée sur cinq sites du Paléolithique supérieur ancien de la vallée de la Vézère (Dordogne, France). 


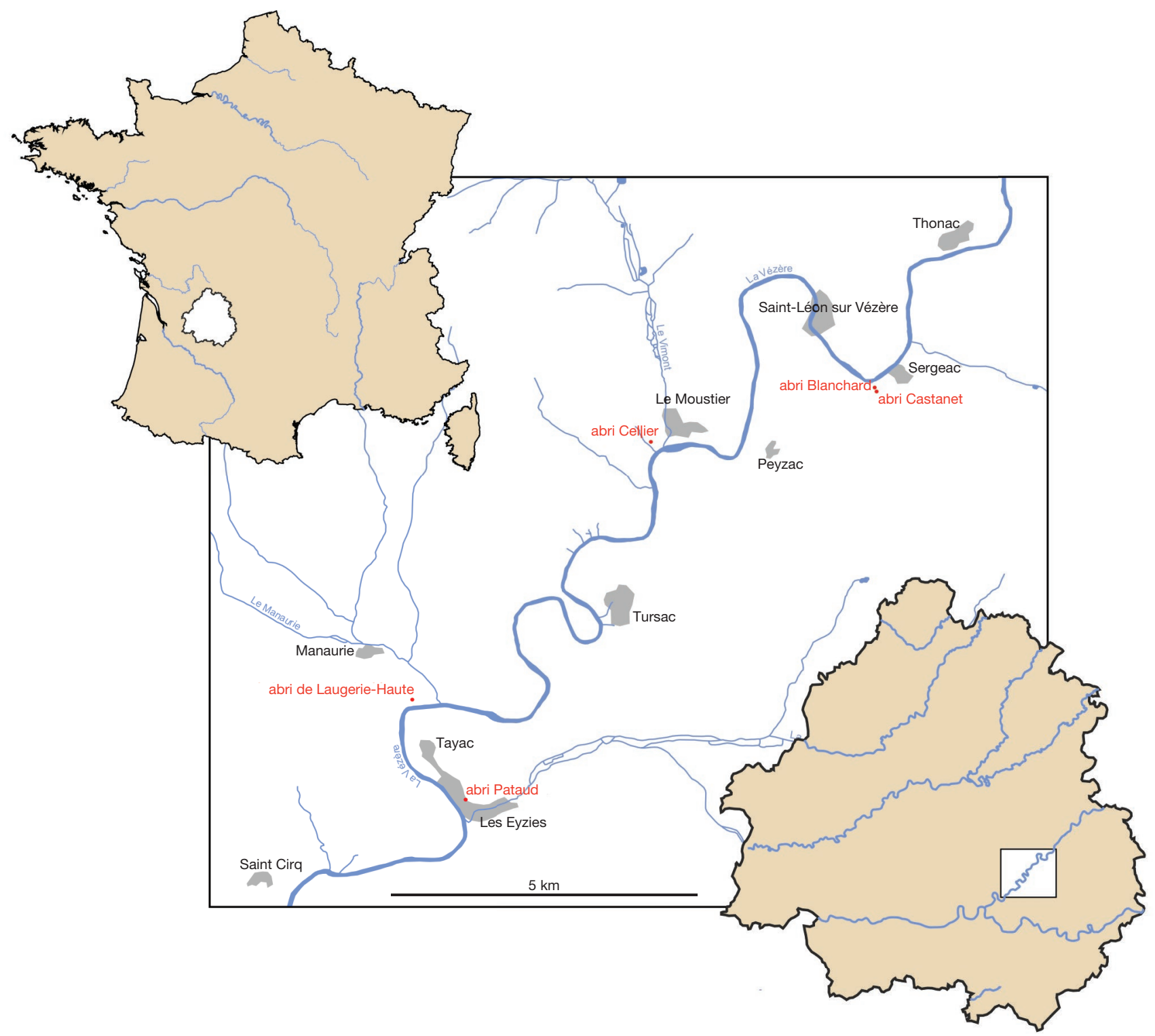

FIG. 1. - Plan de localisation des cinq sites étudiés (DAO L. Chiotti).

Cette étude a été réalisée dans le cadre de plusieurs opérations archéologiques programmées conduites depuis 2005 : "L'occupation humaine de l'abri Pataud il y a 22000 ans", fouille 2005-2015, codirigée par R. Nespoulet et L. Chiotti (Nespoulet et al. 2013) ; "L'abri Castanet, secteur sud", fouille 2005-2010, dirigée par R. White (White 2010) ; «L'abri Blanchard", fouille 2011-2012, dirigée par R. White (White 2012); "L'abri Cellier», fouille 2014, dirigée par R. White (White 2014). Ces matériaux, parfois très nombreux, ont été employés pour des usages extrêmement diversifiés, aussi bien domestiques que symboliques (Chiotti et al. 2018). Parmi eux, de nombreux objets ont été utilisés pour des activités de percussion. Ce sont ces derniers qui font l'objet de la présente étude.

Bien qu'elles soient majorita irement présentes sur des galets de divers matériaux, des traces de percussions sont également observées sur d'autres types de supports, tels que certains objets en silex ayant assumé d'autres fonctions avant de participer à des activités de percussion.
Les sites étudiés se situent tous dans la vallée de la Vézère (Dordogne, France) ou dans de petits vallons tributaires de cette dernière (Fig. 1). Trois d'entre eux ont livré des niveaux d'occupation exclusivement aurignaciens : les abris Blanchard et Castanet dans le vallon de Castel Merle à Sergeac et l'abri Cellier à Tursac. Les deux autres sites, Pataud et Laugerie-Haute, sont de grands abris effondrés présentant des séquences d'occupation beaucoup plus importantes, localisés dans la vallée de la Vézère, sur la commune des Eyzies-de-Tayac. Dans ces deux derniers cas, seuls les niveaux attribués au Gravettien final ont été étudiés.

Les collections étudiées sont conservées à l'abri Pataud, Muséum national d'Histoire naturelle (abri Pataud) et au Musée national de Préhistoire des Eyzies (abris Castanet, Blanchard et de LaugerieHaute). Le matériel de l'abri Cellier provient d'une collection privée (collection Touron) en dépôt au Musée national de Préhistoire. Ces collections ont des origines très diverses, car elles sont issues de fouilles anciennes, de collectes dans les déblais de fouilles anciennes, de fouilles actuelles, ou encore de fouilles clandestines. 

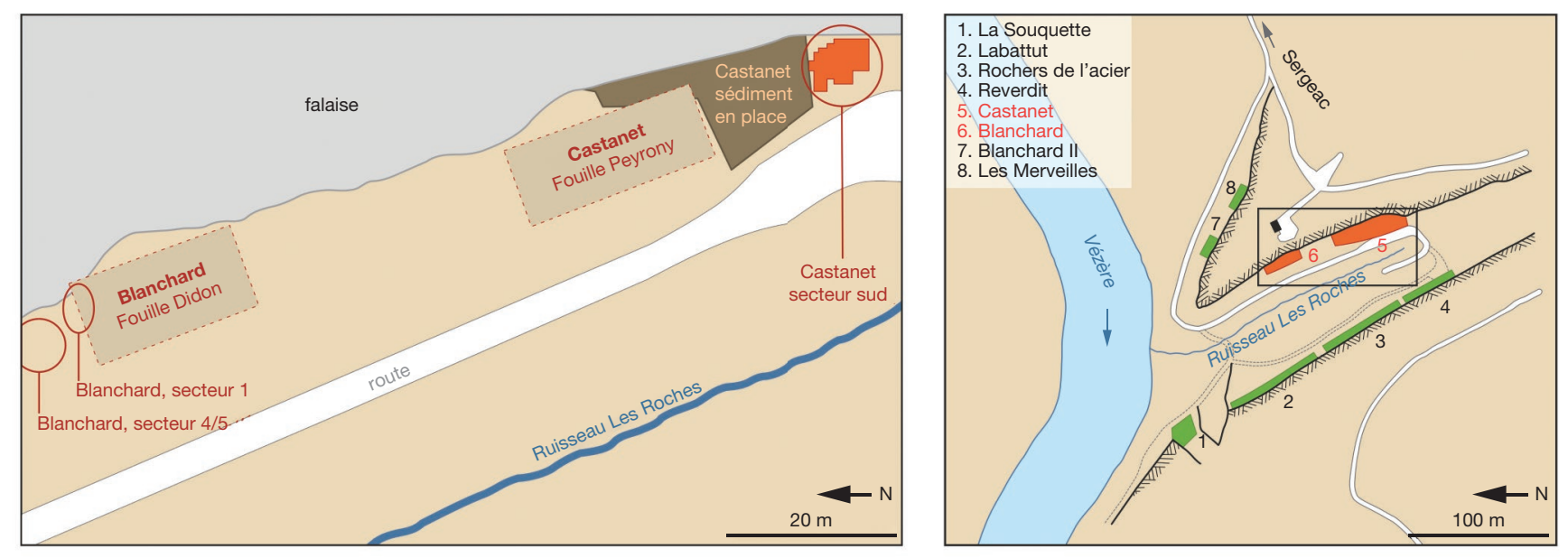

FIG. 2. - Plan du vallon des Roches ou vallon de Castel Merle (d'après Sonneville-Bordes 1960, modifié) et localisation des différents secteurs fouillés dans les abris Castanet et Blanchard (DAO L. Chiotti).

\section{LES SITES AURIGNACIENS}

Les abris Castanet et Blanchard, Sergeac

Les abris voisins Castanet et Blanchard se situent sur la rive droite de la Vézère, le long d'un petit affluent qui porte le nom de vallon des Roches ou vallon de Castel Merle (Fig. 2). Ils s'ouvrent sur une même terrasse rocheuse, perchée une douzaine de mètres au-dessus du vallon et située au pied d'un abrupt rocheux d'environ douze mètres de hauteur.

\section{L'abri Castanet}

L'abri Castanet fut découvert et fouillé par M. Castanet et D. Peyrony entre 1911 et 1913, puis en 1924 et 1925. Ce n'est qu'en 1935 que D. Peyrony publie une coupe stratigraphique et une description du matériel archéologique (Peyrony 1935). Il reconnaît deux niveaux distincts : un niveau inférieur attribué à l'Aurignacien I (ancien) qui repose directement sur le substrat rocheux, et un niveau supérieur attribué à l'Aurignacien II (récent) (Fig. 3).

Si le niveau aurignacien ancien est clairement identifié, l'attribution du niveau supérieur s'avère plus ambiguë, car son industrie lithique se révèle similaire à celle du niveau inférieur (Sonneville-Bordes 1960). L'attribution de ce second niveau à l'Aurignacien récent repose dès lors uniquement sur la présence de quelques sagaies losangiques (Peyrony 1935).

Entre 1994 et 1998, puis entre 2005 et 2010, de nouvelles fouilles sont menées sur la terminaison sud du gisement, respectivement par R. White et J. Pelegrin (Pelegrin $\&$ White 1998), puis par R. White (White 2010). Dans ce secteur, seul le niveau aurignacien ancien, situé au contact du substrat, est reconnu. Plus haut en stratigraphie, quelques pièces sont découvertes au sein d'un éboulis, mais celles-ci sont peu nombreuses et ne permettent pas la reconnaissance d'un véritable niveau archéologique. Ces éléments posent question quant à l'existence de la couche supérieure identifiée par Peyrony : soit cette couche était constituée de matériel en position secondaire comme c'est le cas au sud, soit un niveau supérieur était bien présent latéralement.
Les collections de MLHS de l'abri Castanet analysées dans le cadre de cette étude ont des origines diverses:

1) La collection Peyrony, conservée au Musée national de Préhistoire, comporte quelques pièces en MLHS, mais ces derniers ont été très peu collectés. Seuls quelques objets en matériaux particuliers tels que des schistes ou de petits galets de calcaire, ou des outils très reconnaissables tels que des percuteurs sont présents. Ce matériel n’est ni coordonné, ni localisé sur la surface de la fouille. Seule sa provenance stratigraphique est précisée.

2) De nombreux MLHS ont été collectés dans les déblais des fouilles Peyrony lors des fouilles 1994-1998 et 2005-2010. Lorigine stratigraphique de ce matériel est indéterminable.

3) Bien qu'en quantités relativement faibles, les fouilles 1994-1998 et 2005-2010 ont également livré des pièces en MLHS percutées.

4) Une série issue d'une fouille clandestine des années $1960 \mathrm{a}$ pu être récupérée lors des fouilles 2005-2010. Bien que les MLHS aient été très peu collectés par le fouilleur clandestin, cette série contient néanmoins quelques pièces intéressant la présente étude.

Au total, 703 pièces provenant de l'abri Castanet ont été examinées, parmi lesquelles 72 présentent des traces de percussions (Tableau 1).

\section{L'abri Blanchard}

L'abri Blanchard fut découvert par A. Reverdit dans les années 1870, mais ne fut fouillé qu'en 1910 et 1911 par L. Didon et M. Castanet (Didon 1911).

Lors de cette fouille, L. Didon reconnait deux niveaux archéologiques : le niveau inférieur B attribué à l'Aurignacien I et situé sur le substrat de la terrasse, et le niveau supérieur $\mathrm{D}$ attribué à l'Aurignacien II (Fig. 4A).

En 2011 et 2012 de nouveaux travaux sont entrepris sous la direction de R. White (White 2011, 2012). Après un siècle d'abandon, de très importants déblais, pour la plupart constitués de déchets récents s'étaient accumulés dans ce secteur. Lélimination de ces déchets et l'enlèvement des importants déblais des fouilles anciennes ont permis de mettre au jour 
A

C : Aurignacien II (récent)

$B$ : niveau stérile

A : Aurignacien I (ancien)
B

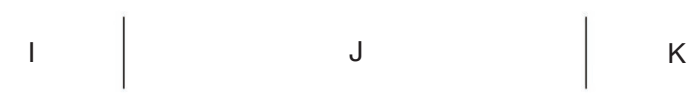

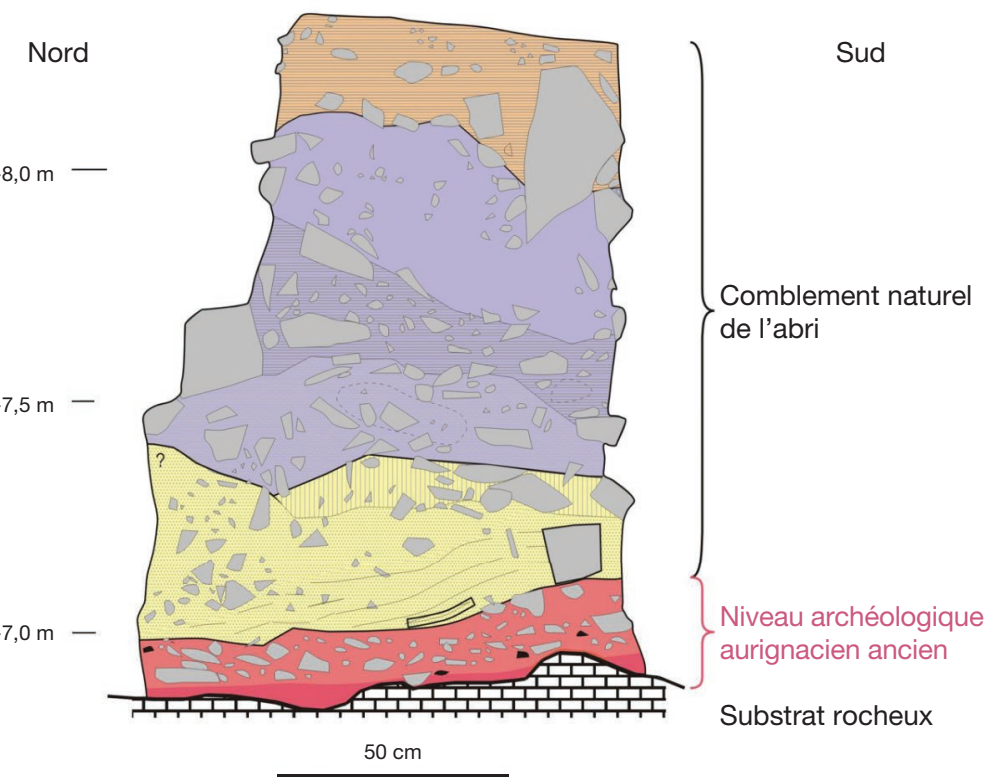

FIG. 3. - Coupes stratigraphiques de l'abri Castanet: A, coupe transversale de la fouille Peyrony (secteur nord; d'après Peyrony 1935); B, coupe longitudinale du secteur sud, fouille White, 2005-2010 (d'après White 2006; DAO Ph. Gardère).
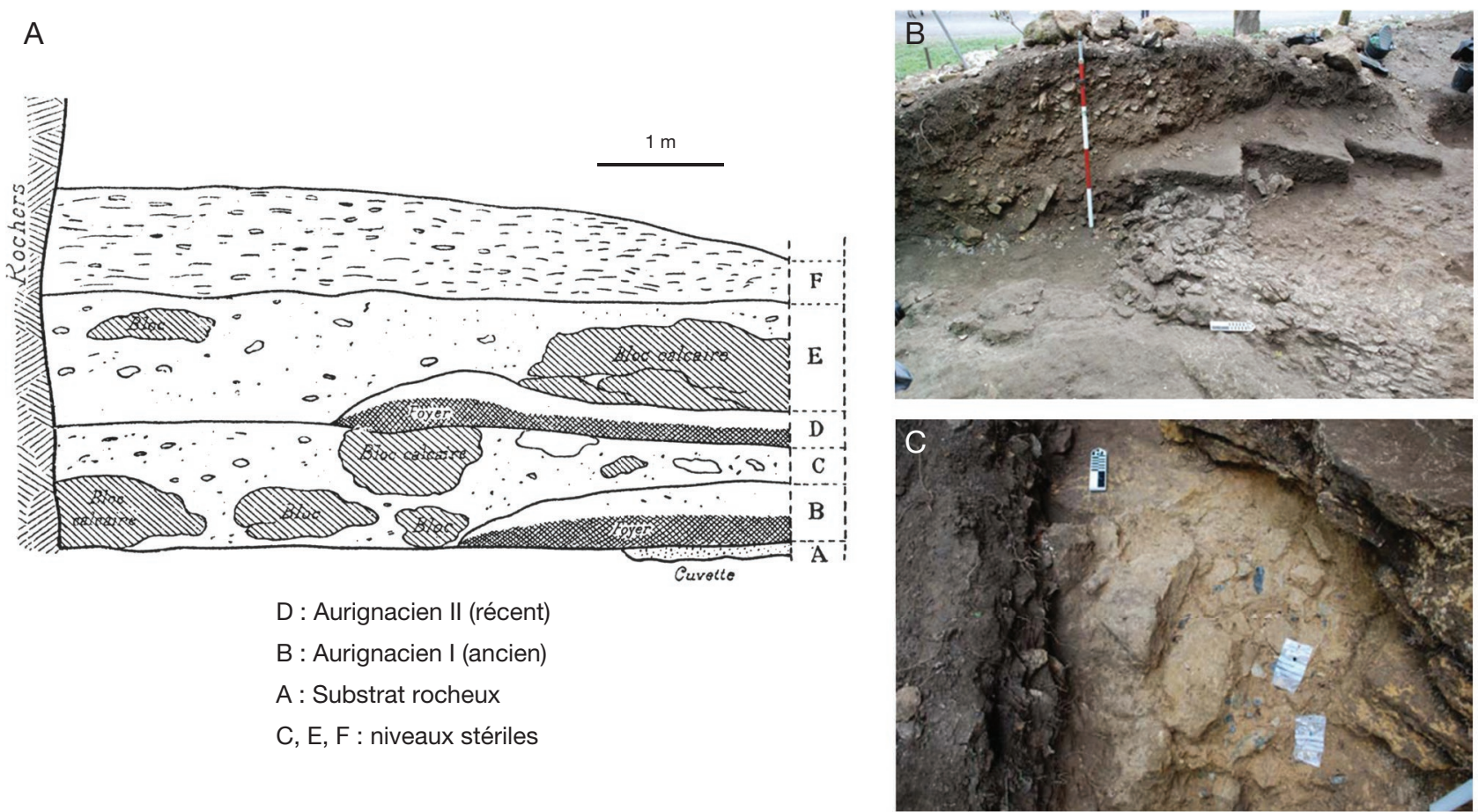

FIG. 4. - Abri Blanchard: A, coupe stratigraphique de la fouille Didon (d'après Didon 1911); B, fouille White, 2011-2012, secteur 4/5, Aurignacien ancien; C, fouille White, 2011-2012, secteur 1, Aurignacien récent (clichés : R. Mensan).

deux lambeaux de couches en place, dénommés secteur 1 et secteur 4/5 (Figs 2;4). Ces deux lambeaux de niveaux non stratifiés ont pu être attribués à l'Aurignacien récent pour le secteur 1 et à l'Aurignacien ancien pour le secteur $4 / 5$ (Chiotti et al. 2015). Il est probable qu'ils correspondent respectivement aux niveaux D et B de L. Didon. 
TABLEAU 1. - Inventaire des MLHS et des MLHS percutés dans l'ensemble des collections étudiées sur les cinq sites.

\begin{tabular}{|c|c|c|c|c|}
\hline Site & Série & Attribution culturelle & Objets percutés & MLHS \\
\hline \multirow[t]{4}{*}{ Blanchard } & fouille White (2011-2012) - Secteur 1 & Aurignacien récent & 5 & 64 \\
\hline & fouille White (2011-2012) - Secteur $4 / 5$ & Aurignacien ancien & 8 & 136 \\
\hline & déblais des fouilles Didon & Aurignacien lato sensu & 60 & 477 \\
\hline & total Blanchard & & 73 & 677 \\
\hline \multirow[t]{6}{*}{ Castanet } & fouille Peyrony (1911-1925) & Aurignacien lato sensu & 28 & 80 \\
\hline & déblais des fouilles Peyrony & Aurignacien lato sensu & 32 & 253 \\
\hline & fouille clandestine (années 1960) & Aurignacien lato sensu & 2 & 19 \\
\hline & fouilles Pelegrin/White (1994-1998) - Secteur sud & Aurignacien ancien & 1 & 77 \\
\hline & fouilles White (2005-2010) - Secteur sud & Aurignacien ancien & 9 & 274 \\
\hline & total Castanet & & 72 & 703 \\
\hline Cellier & fouilles White (2014) & Aurignacien lato sensu & 10 & 61 \\
\hline \multirow[t]{4}{*}{ Pataud } & fouille Movius (1958-1963) & Gravettien final & 34 & 99 \\
\hline & fouille clandestine (années 1970-1980) & Gravettien final & 14 & 261 \\
\hline & fouille Nespoulet/Chiotti (2005-2015) & Gravettien final & 27 & 640 \\
\hline & total Pataud & & 75 & 1000 \\
\hline \multirow[t]{4}{*}{ Laugerie-Haute Est } & fouille Peyrony (1927-1935) - couche F & Gravettien final & 19 & 53 \\
\hline & fouille Bordes (1955-1960) - couche 36 & Gravettien final & 20 & 339 \\
\hline & fouille Bordes (1955-1960) - couche 38 & Gravettien final & 6 & 31 \\
\hline & total Laugerie-Haute Est & & 45 & 423 \\
\hline Total & & & 275 & 2864 \\
\hline
\end{tabular}

Comme pour l'abri Castanet, les collections de MLHS de l'abri Blanchard qui ont été étudiées ont des origines différentes. Suite aux fouilles de L. Didon, les collections de l'abri Blanchard ont été dispersées dans de nombreuses institutions à travers le monde (Delluc \& Delluc 1981; Tartar et al. 2014). De ce fait, seules les collections constituées lors des fouilles récentes ont été étudiées. Elles comprennent :

1) le matériel issu de la fouille des deux lambeaux du secteur 1 et du secteur 4/5;

2) le matériel collecté sur le talus, dans les déblais des fouilles Didon.

Un total de 677 objets en MLHS a été décompté pour l'abri Blanchard, dont 73 outils portant des stigmates dérivant d'une activité de percussion (Tableau 1).

\section{L'abri Cellier, Tursac}

L'abri Cellier est un abri-sous-roche effondré situé sur le promontoire qui sépare la vallée du Vimont et la combe de Vergne sur la commune de Tursac. Orienté au sud-est, il est creusé dans le coteau calcaire dominant la Vézère sur sa rive droite.

En 1905, D. Peyrony fut le premier à fouiller l'abri Cellier. Le site a ensuite été sondé en 1926 par F. Merlan, puis fouillé en 1927 par G. Collie et P. Nesbitt (Collie 1928; Peyrony 1946) pour le compte du Logan Museum of Anthropology. D'après les documents laissés par les fouilleurs, l'abri Cellier a livré deux niveaux archéologiques aurignaciens : un niveau aurignacien ancien, situé en contact direct avec le substrat, et un niveau aurignacien récent, séparé du précédent par une couche stérile (Fig. 5). La présence et l'attribution d'un troisième niveau plus haut dans la stratigraphie ne sont pas claires : s'agit-il vraiment d'un niveau archéologique, ou de matériel dérivé du plateau, et à quelle période ce matériel doit-il être attribué (Aurignacien récent ou Gravettien; Peyrony 1946)?
En 2014, suite à une analyse des archives et à une inspection préliminaire des lieux, R. White a entrepris une nouvelle exploration du site à la tête d'une équipe pluridisciplinaire. Cette opération visait à identifier d'éventuels dépôts archéologiques préservés dans le site et d'en déterminer, le cas échéant, la composition, dans le but d'y reprendre des fouilles. Ces travaux ont révélé que l'abri avait été presque entièrement vidé lors des fouilles anciennes. Ils ont cependant permis de confirmer la présence de l'Aurignacien ancien directement sur le substrat rocheux (White 2014). L’Aurignacien récent est bien attesté dans les déblais des fouilles Collie, mais n'a pas été retrouvé en place. Enfin, aucune trace de Gravettien n'a été retrouvée dans les déblais. Il est donc très probable que le «niveau » supérieur soit en position secondaire dans les éboulis et qu'il soit également aurignacien (White 2014).

La collection issue des fouilles Collie est conservée en grande majorité au Logan Museum of Anthropology, Beloit College (Wisconsin, États-Unis) et pour une petite partie au Musée national de Préhistoire. Ces collections n'ont pas pu être examinées jusqu'à présent, aussi la présence d'objets en MLHS en leur sein demeure incertaine.

Seul le matériel issu de la fouille des rares lambeaux de couches mis au jour en 2014 est intégré dans cette étude. Bien qu'extrêmement riche en MLHS, le matériel issu des déblais des anciennes fouilles n'a pas encore pu être étudié.

Actuellement, seules 61 pièces provenant de l'abri Cellier ont donc été analysées, parmi lesquelles dix seulement ont servi de percuteurs (Tableau 1).

Pour l'ensemble des trois sites, du fait de l'hétérogénéité et de l'origine des différentes collections, il n'a pas été possible de séparer les différents niveaux. Nous considérerons donc globalement les séries de chacun de ces sites. Étant donné qu'il s'agit uniquement de niveaux aurignaciens, cette approximation nous a paru acceptable dans le cadre de cette étude. 
A

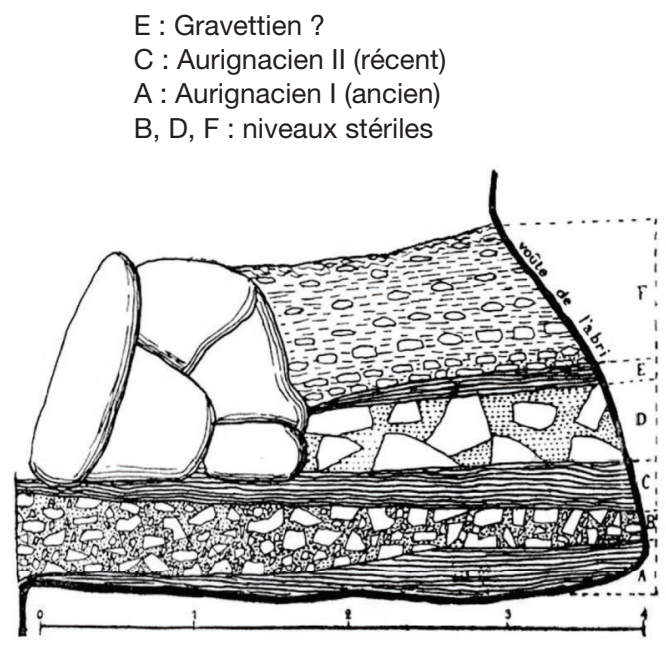

B

US 100 : niveau indéfini en position secondaire (Aurignacien probable) US 102 : niveau noir indéfini (probable Aurignacien récent) US 104 : niveau de base, Aurignacien ancien US 00, 01, 101 et 103 : unités stériles

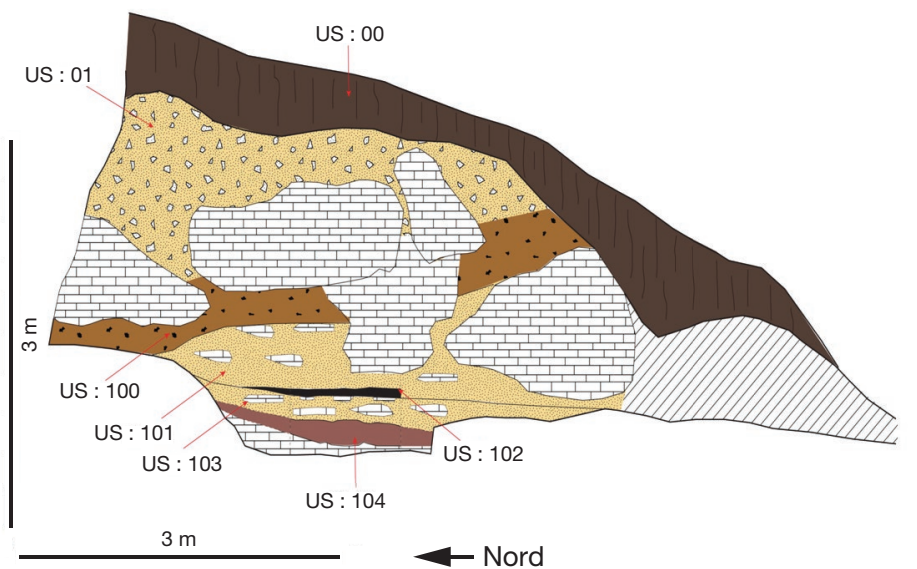

FIG. 5. - Coupes stratigraphiques de l'abri Cellier : A, coupe de la fouille Collie, 1927 (d'après Peyrony 1946); B, coupe de la fouille White, 2014, située du côté est du site, dans la terminaison de l'abri, où il ne reste quasiment plus de dépôts conservés (d'après White 2014; DAO R. Mensan).

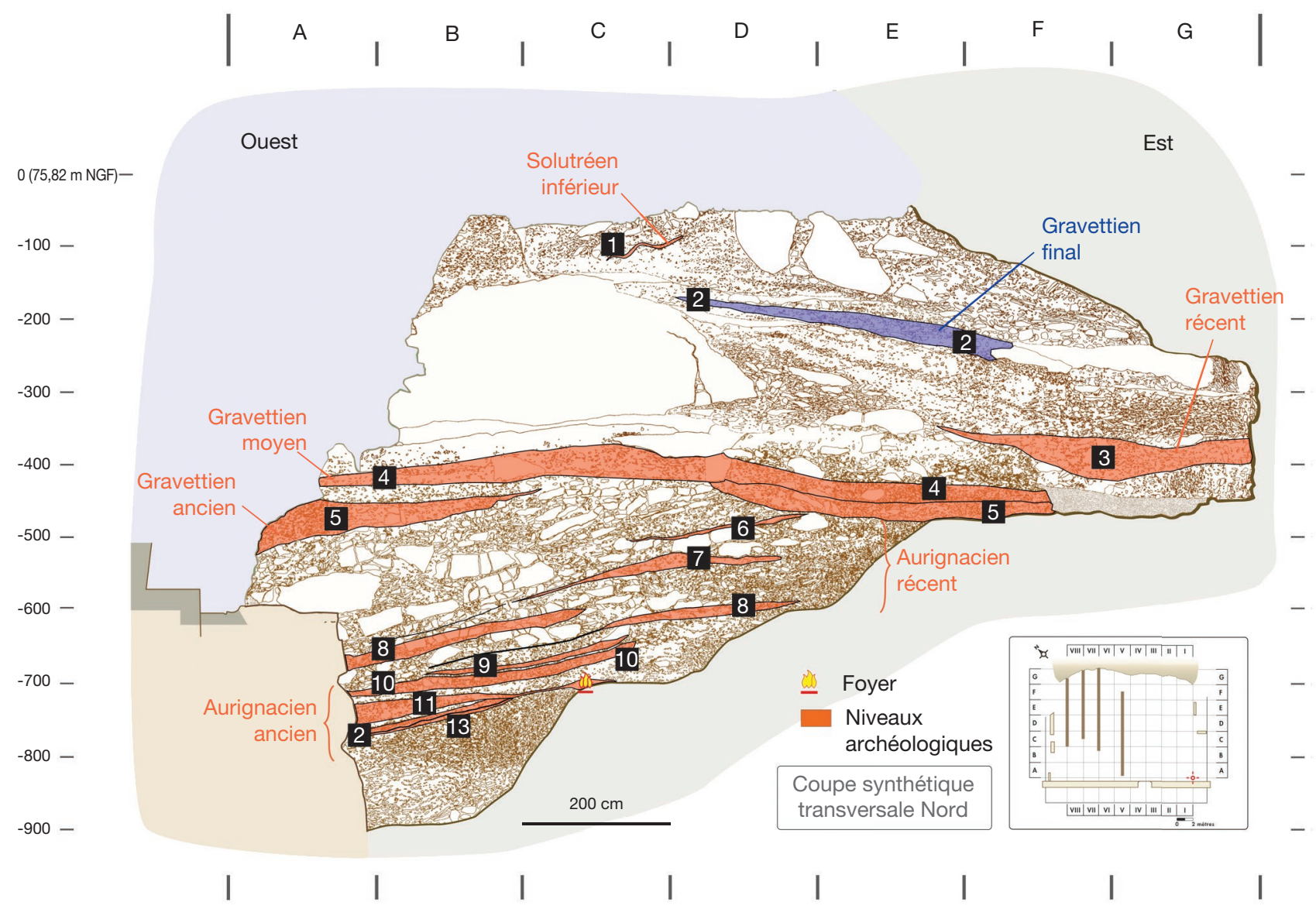

FIG. 6. - Coupe stratigraphique synthétique transversale nord de l'abri Pataud, fouille Movius, 1958-1964 (DAO C. Lecante). 


\section{LES SITES DU GRAVETTIEN FINAL}

\section{L'ABri Pataud, Les EyzIES-DE-TAyaC}

Situé dans le village des Eyzies-de-Tayac, l'abri Pataud se trouve au pied d'une falaise de calcaire d'une trentaine de mètres de hauteur, sur la rive gauche de la Vézère. Il s'agit d'un ancien abri-sous-roche totalement effondré, situé une vingtaine de mètres au-dessus du lit actuel de la Vézère.

Le site qui appartient au Muséum national d'Histoire naturelle a été fouillé entre 1958 et 1964 par H. L. Movius (1975, 1977). Ces fouilles ont permis de mettre au jour 14 couches d'occupation réparties dans un remplissage de 9,25 mètres d'épaisseur (Fig. 6).

La stratigraphie définie par H. L. Movius comprend neuf niveaux aurignaciens (couches 14 à 6 ), quatre niveaux gravettiens (couches 5 à 2) et un niveau solutréen inférieur (couche 1).

Entre 2005 et 2015, une nouvelle fouille, codirigée par R. Nespoulet et L. Chiotti, a été menée sur la couche 2 attribuée au Gravettien final (Nespoulet et al. 2013).

Les MLHS présentés ici ont été étudiés dans le cadre du projet de recherche mené de 2005 à 2015; ceux-ci proviennent donc exclusivement du niveau daté du Gravettien final (Chiotti et al. 2018). Comme pour les sites précédents, le matériel examiné a plusieurs origines. Les pièces issues de la collection Movius sont assez peu nombreuses, car l'attribution stratigraphique d'une grande partie des MLHS non coordonnés et non marqués a été perdue (Chiotti et al. 2018). En conséquence, les objets étudiés provenant des fouilles Movius sont tous des objets dont les coordonnées ont été relevées lors de la fouille. Le matériel issu de la fouille 2005-2015 est entièrement coordonné et contrairement à celui de la fouille Movius, il comprend également des refus de tamis. Enfin, une troisième série provenant des déblais d'une fouille clandestine a été étudiée. Malgré les circonstances de sa collecte, cette série peut être attribuée avec certitude au Gravettien final dans la mesure où la destruction a affecté exclusivement la couche 2 . Mille pièces en MLHS ont été décomptées dans le mobilier du niveau gravettien final de l'abri Pataud, parmi lesquelles 75 sont liées à des activités de percussion (Tableau 1).

\section{L'ABRI DE LAUgerie-Haute, LES EyZIES-DE-TAYAC}

Situé à environ deux kilomètres en amont du village des Eyzies-de-Tayac, sur la rive droite de la Vézère, le site de Laugerie-Haute comprend deux locus séparés par un témoin central : Laugerie-Haute Est et Laugerie-Haute Ouest. Malgré les nombreuses fouilles réalisées depuis la découverte de l'abri en 1862, il faut attendre les travaux de D. et E. Peyrony, entre 1927 et 1935 , pour que sa stratigraphie soit vraiment établie (Peyrony \& Peyrony 1938).

Notre étude concerne uniquement le Gravettien final qui n'est présent que dans locus Est. Ce dernier, comporte une stratigraphie allant du Gravettien récent au Magdalénien supérieur, en passant par une séquence solutréenne comptant parmi les plus complètes connues sur le territoire français (Peyrony \& Peyrony 1938; Bordes 1958).

Entre 1955 et 1960, le locus Est a été réinvesti par des fouilles dirigées par F. Bordes (Bordes 1958).
Le niveau concerné par la présente étude est la couche $\mathrm{F}$ de D. Peyrony, qui a été attribuée par cet auteur au «Protomagdalénien ", terme correspondant dans la terminologie actuelle au Gravettien final. Au cours de ses fouilles, F. Bordes a également retrouvé du matériel "protomagdalénien " au sein d'un niveau principal (couche 36) et d'un niveau secondaire (couche 38) interprétés comme un dédoublement du niveau $\mathrm{F}$ de D. Peyrony, dédoublement qui apparaissait déjà sur la coupe relevée par ce dernier (Fig. 7; Bordes 1978).

Les collections des fouilles Peyrony et des fouilles Bordes sont toutes deux conservées au Musée national de Préhistoire. Elles témoignent ici aussi de la mise en ouvre de stratégies de fouilles très différentes par ces deux auteurs.

Quatre-cent-vingt-trois pièces en MLHS ont été analysées, parmi lesquelles 45 concernent du matériel de percussion (Tableau 1).

\section{MATÉRIEL ET MÉTHODES}

\section{DÉFINITIONS}

La détermination des MLHS portant des traces de percussions a été effectuée par une analyse macroscopique et/ou par des observations à la loupe binoculaire. Ces objets se répartissent en huit catégories typologiques :

\section{1) Des percuteurs classiques ou percuteurs de taille (utilisés pour le débitage du silex)}

Les percuteurs classiques sont définis comme étant «en général des galets de rivière [ayant] une forme régulière sphérique, ovoïde ou oblongue et des angles arrondis. Leurs stigmates d'utilisation sont des traces d'impacts et d'écrasement pouvant aller jusqu'à de véritables enlèvements sur les parties contondantes (angles, arêtes ou extrémités)» (Beaune 2000 : $50)$. Ces galets ne sont en général pas aménagés. J. Tixier les décrit comme un "marteau naturel utilisé pour débiter ou retoucher la pierre dure» (Tixier et al. 1980 : 96). Selon les auteurs, ils peuvent également être nommés percuteurs de taille (Soressi et al. 2008; Daulny \& Dachary 2009).

\section{2) Des percuteurs dits de concassage}

Les percuteurs de concassage sont des galets ayant subi des percussions beaucoup plus violentes. Ils présentent en conséquence d'intenses traces d'impacts sur les extrémités ou les arêtes, traces qui peuvent prendre la forme de véritables enlèvements modifiant parfois la morphologie et le volume du support (Beaune 1993a, 2000; Beaune \& Santallier 2003).

L'étude des percuteurs de concassage du site azilien de Pont d'Ambon (Bourdeilles, Dordogne) a permis d'émettre l'hypothèse que ces outils étaient probablement utilisés pour fragmenter de l'os (Célérier \& Kervazo 1988), et qu'ils participaient donc sans doute à des activités de boucherie sur de gros animaux (concassage des os pour l'extraction de la moelle; Beaune 1993a, 2000). Des expérimentations menées par le Dr Henri-Martin dès le début du XXe siècle avaient d'ailleurs déjà montré que de tels galets convenaient parfaitement pour fracturer des os longs de cheval (Henri-Martin 1910). 
A

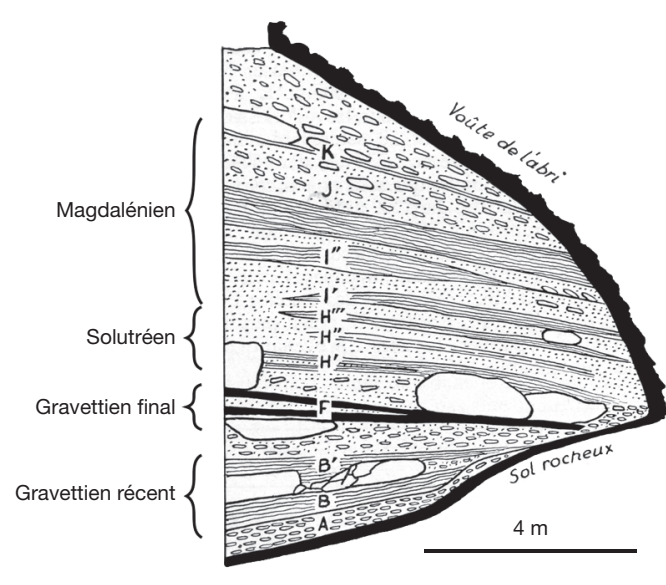

B

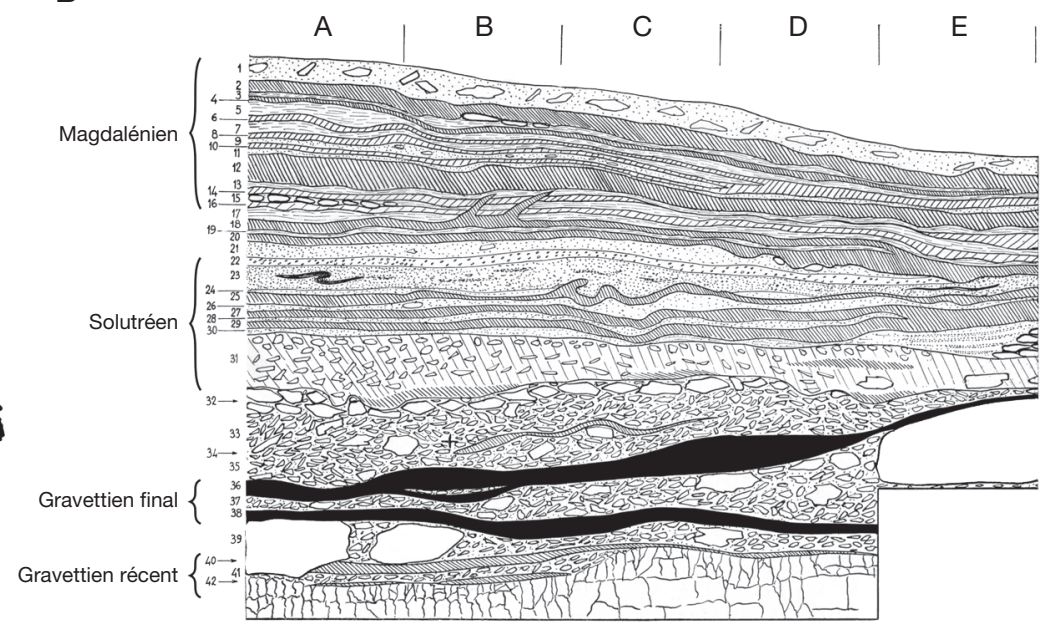

FIG. 7. - Coupes stratigraphiques de l'abri de Laugerie-Haute Est :A, coupe de la fouille Peyrony (d'après Peyrony \& Peyrony 1938); B, coupe de la fouille Bordes (d'après Bordes 1958).

Selon les auteurs, ces outils apparaissent également sous le terme de percuteurs de fracturation (Daulny 2006b; Soressi et al. 2008; Daulny \& Dachary 2009).

\section{3) Des choppers (dont les arêtes ont été utilisées comme percuteurs)} Les choppers «sont des outils façonnés sur galets roulés ou blocs anguleux, dégageant un tranchant rectiligne ou plus ou moins sinueux et généralement partiel» (Chavaillon in LeroiGourhan 1997). Bien que caractéristiques des périodes très anciennes (premières industries humaines), ces objets perdurent jusqu’au Néolithique (Chavaillon in Leroi-Gourhan 1997).

Dans certaines industries du Paléolithique supérieur, on en rencontre parfois des exemplaires, souvent sur galets de quartz, qui portent de très importantes traces d'écrasement et de percussions couvrant la majeure partie des arêtes. La plupart d'entre eux portent également sur leurs surfaces néocorticales de fortes plages de traces de percussions, très similaires à celles observées sur les percuteurs de concassage (Chiotti et al. 2018). Ces éléments nous incitent à penser que ces choppers ont pu être utilisés pour des usages similaires à ceux des percuteurs de concassage (Chiotti et al. 2018).

\section{4) Des percuteurs de pierre tendre}

Les percuteurs minéraux sont qualifiés de percuteurs de pierre tendre ou percuteurs «"dur-tendre" lorsqu'il s'agit de roches de plus faible densité [que les galets de quartz, quartzite, granite ou basalte constituant généralement les percuteurs dits "durs»] ou poreuse du type grès ou calcaire» (Aubry in Vialou 2004).

Selon J. Pelegrin, "les percuteurs de pierre tendre les plus efficaces sont des galets, ou "pavés" à sommets arrondis de grès fin médiocrement cimenté, à contact sableux [...]. Il peut aussi s'agir de nodules de silex ovoïdes à cortex épais, c'est alors le cortex qui joue le rôle de pierre tendre, mais la durée d'emploi de tels percuteurs est plus faible» (Pelegrin 2000 : 78).

Les percuteurs de pierre tendre sont relativement rares dans les sites du Paléolithique supérieur, ce qui est probablement à mettre en relation avec le matériau utilisé (souvent le calcaire encaissant des abris) qui a pu les rendre difficiles à identifier ou peu dignes d'intérêt, en particulier lors des fouilles anciennes.

\section{5) Des petits galets présentant des traces d'utilisation diversifiées (notamment d'utilisation comme maillets)}

Des galets relativement petits, généralement allongés, portent sur leurs surfaces de nombreuses traces d'utilisation, souvent très variées. Celles-ci comprennent des facettes d'abrasion, des traces d'impacts isolées ou regroupées en plages, des traces de percussions sur les arêtes, des stries isolées ou regroupées en faisceaux ou en plages, des polis d'utilisation et des traces de raclage, voire des gravures (Chiotti et al. 2018).

Certains de ces galets portent notamment des traces d'impacts sur leurs faces planes qui forment des concentrations plus ou moins denses, créant parfois de nettes dépressions dans la surface du galet. Il est fréquent de trouver plusieurs de ces concentrations sur une même pièce. Elles se situent en général au niveau des extrémités. Ces traces, ainsi que le gabarit des galets utilisés, correspondent aux caractéristiques retenues par S. de Beaune pour définir les maillets : "Ce sont de petits galets plats et allongés, portant des traces d'impacts sur une ou les deux extrémités de leurs surfaces, parfois suffisamment intenses et nombreuses pour former une véritable cupule" (Beaune 2000 : 58). L'auteure fait ainsi l'hypothèse que ces outils ont pu être utilisés comme «de petits maillets travaillant en percussion légère indirecte» (Beaune 1997, 2000).

\section{6) Des pièces en silex réutilisées comme percuteurs (nucléus, grattoirs carénés, éclats)}

Même si J. Tixier et al. signalent leur existence dans leur définition des percuteurs : «certains objets taillés ont parfois été utilisés comme percuteurs" (Tixier et al. 1980), les objets en silex taillés réutilisés comme percuteurs sont relativement peu fréquents dans la littérature. Au mieux, ils sont simplement cités lors de la description des industries lithiques. Ce sont des objets de dimensions variables, le plus souvent d'anciens nucléus. Il peut s'agir de pièces présentant de petites plages de 
traces de percussions localisées, jusqu'à des pièces couvertes de traces de percussions qui éliminent presque complètement les arêtes de la pièce d'origine.

Une étude expérimentale réalisée afin de déterminer la fonction de pièces similaires datées du Paléolithique moyen a permis de montrer qu'une utilisation comme percuteur ou retouchoir pour la taille du silex est susceptible de laisser des stigmates comparables à ceux observés sur les pièces étudiées (Claud et al. 2010; Thiébaut et al. 2010). Cependant, ce type de percuteur n'est pas le plus adapté pour ce type d'activité, un galet permettant à la fois une meilleure préhension et une plus grande précision (Thiébaut et al. 2010).

L'hypothèse d'une utilisation comme bouchardes peut également être envisagée. Le bouchardage consiste «à porter, avec un percuteur de pierre, des chocs répétés qui déterminent de petits écrasements sur la pièce travaillée» (Pelegrin in LeroiGourhan 1997). Sur la base d'expérimentations et d'analyses de pièces archéologiques, C. Sestier et C. Bontemps, se demandent «si de tels objets n'étaient pas plutôt des percutants pour le façonnage de surfaces de pierre, donc des bouchardes" (Sestier \& Bontemps 2003 : 308). Des blocs de calcaire gravés et sculptés sont présents dans les différents sites étudiés, en particulier dans les sites aurignaciens (White et al. 2012, 2018; Bourrillon et al. 2018). Les pièces en silex percutées présentes sur ces sites pourraient correspondre à des bouchardes utilisées pour la réalisation de ces gravures, notamment lors des phases de préparation et de régularisation préalable de la surface des blocs.

Une troisième hypothèse est celle d'un usage comme briquets. Des approches expérimentales et analytiques ont en effet permis à Th. Morala et M. Baillet (communication personnelle de Th. Morala, travaux en cours) de montrer qu'un usage répété en percussion sur de la pyrite ou de la marcassite laissait des traces similaires à celles observées sur certains de ces objets, en particulier ceux montrant de forts polis sur leurs faces et leurs arêtes.

\section{7) Des pièces intermédiaires}

Les pièces classées dans cette catégorie sont des objets en silex taillés, réemployés pour des activités ayant laissé des stigmates de percussion. Contrairement à la catégorie précédente, ces stigmates se localisent toujours selon deux pôles opposés du support.

Le caractère strictement simultané de la formation de ces stigmates n'étant pas démontrable, il est possible d'envisager que ceux-ci puissent correspondre à des percuteurs disposant de deux parties actives utilisées successivement. Cependant, la position des traces, systématiquement situées sur deux pôles opposés de ces objets, laisse plutôt supposer un usage en tant que pièces intermédiaires entre le percuteur et le matériau à travailler. Étant donné la nature des traces observées, il est probable que cet usage soit proche de celui des pièces esquillées en silex (Le Brun-Ricalens 2006). Les principales différences entre ces dernières et les outils examinés concernent les dimensions des supports en silex sélectionnés (beaucoup plus volumineux pour les objets étudiés ici que ceux des pièces esquillées) et l'intensité de leur utilisation (généralement beaucoup plus intense pour les pièces esquillées).

\section{8) Des enclumes}

Selon S. de Beaune, "les objets dont l'utilisation comme enclume est plausible sont les galets, blocs ou plaquettes présentant des traces d'impacts sur une ou plusieurs faces mais également parfois aussi sur leurs arêtes. [...] L'enclume peut avoir servi de support à n'importe quel geste de percussion lancée. Ses deux principaux usages sont le débitage du silex et le concassage de matériaux durs tels que des os ou des écales de noix" (Beaune 2000 : 46). Ces objets sont parfois dénommés "percuteur passif» (Chavaillon 1979) ou encore «percuteur dormant» (Tixier et al. 1980).

\section{REPRÉSENTATIVITÉ DES COLLECTIONS}

Avant de présenter les objets présents dans les collections étudiées, il convient de rappeler que ces dernières ont des origines très diverses (fouilles anciennes, fouilles récentes, déblais, fouilles clandestines) et que par conséquent elles n'ont pas toutes la même valeur informative. Malgré leur disparité liée à des périodes (et donc à des techniques) de fouille différentes, les séries gravettiennes proviennent toutes de niveaux bien identifiés, de la partie finale de ce technocomplexe. Au contraire pour les trois sites aurignaciens, les séries proviennent à chaque fois de différents niveaux, qui bien que tous aurignaciens, n'ont pas toujours pu être distingués au sein des collections, elles ne sont, pour la plupart, pas exhaustives et nous n'avons pas eu accès à l'ensemble des collections anciennes.

Par conséquent, les possibilités de comparaison et d'interprétation des données entre les deux périodes restent limitées.

\section{MATÉRIEL}

Deux-cent-soixante-quinze objets portant des traces de percussions ont pu être identifiés dans les collections des cinq sites.

À l'exception de l'abri Castanet où ils arrivent en seconde position, les objets les plus nombreux sont toujours les percuteurs destinés à la taille du silex (Tableau 2).

Par ordre d'importance quantitative, viennent ensuite les percuteurs de concassage, qui s'avèrent cependant beaucoup mieux représentés sur les sites aurignaciens que sur les sites gravettiens final. Il en va de même pour les choppers/percuteurs, qui comme nous l'avons dit plus haut, ont un usage probablement similaire aux percuteurs de concassage.

Contrairement aux deux catégories précédentes, les nucléus en silex réutilisés comme percuteurs sont plus fréquents sur les sites du Gravettiens final. Ils se révèlent même nombreux à l'abri Pataud. Dans les sites aurignaciens, ces nucléus sont parfois des grattoirs carénés.

Outre ces catégories les plus représentées, on décompte encore vingt petits galets portant des plages de traces de percussions très marquées et bien localisées, qui peuvent être considérés comme des maillets (Beaune 2000). Les percuteurs de pierre tendre sont des objets beaucoup plus rares : on en dénombre seulement trois exemplaires. Le site de Castanet a livré de grosses pièces en silex probablement réutilisées en pièces intermédiaires. Enfin, des objets utilisés comme enclumes sont recensés dans les cinq sites. 
TABLEAU 2. - Inventaire du matériel de percussion identifié sur les cinq sites étudiés. Pour les enclumes, le nombre entre parenthèses représente le nombre total de pièces identifiées, c'est-à-dire le nombre d'enclumes (nombre hors parenthèses) additionné au nombre d'enclumes ayant assumé d'autres fonctions et qui, pour cette raison, sont décomptées dans d'autres colonnes du tableau.

\begin{tabular}{|c|c|c|c|c|c|c|c|c|c|c|c|c|}
\hline & & 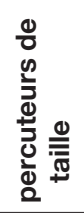 & 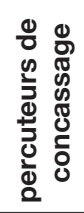 & 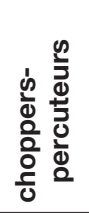 & 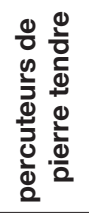 & 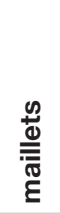 & 兽 & 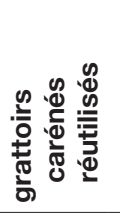 & 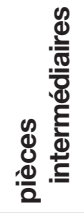 & $\begin{array}{l}\stackrel{g}{E} \\
\frac{E}{5} \\
\frac{J}{0} \\
\frac{E}{0}\end{array}$ & 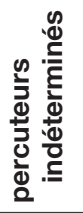 & $\begin{array}{l}\text { तु } \\
\stackrel{0}{0}\end{array}$ \\
\hline \multirow[t]{2}{*}{ Gravettien final } & Pataud & 29 & 5 & 2 & 1 & 10 & 26 & 0 & 0 & $1(2)$ & 1 & 75 \\
\hline & Laugerie-Haute & 14 & 5 & 4 & 0 & 5 & 9 & 0 & 0 & $0(1)$ & 8 & 45 \\
\hline \multirow[t]{3}{*}{ Aurignacien } & Blanchard & 22 & 16 & 11 & 2 & 0 & 0 & 0 & 0 & $8(13)$ & 14 & 73 \\
\hline & Castanet & 15 & 16 & 4 & 0 & 4 & 6 & 6 & 9 & $2(8)$ & 10 & 72 \\
\hline & Cellier & 2 & 1 & 1 & 0 & 1 & 1 & 1 & 0 & $1(1)$ & 2 & 10 \\
\hline Total & & 82 & 43 & 22 & 3 & 20 & 42 & 7 & 9 & $12(25)$ & 35 & 275 \\
\hline
\end{tabular}

TABLEAU 3. - Répartition des matériaux utilisés en percussion par site et par culture.

\begin{tabular}{|c|c|c|c|c|c|c|c|c|c|c|c|}
\hline \multirow[b]{2}{*}{ Matière } & \multirow[b]{2}{*}{ Blanchard } & \multirow[b]{2}{*}{ Castanet } & \multirow[b]{2}{*}{ Cellier } & \multirow[b]{2}{*}{ Pataud } & \multirow{2}{*}{$\begin{array}{c}\text { Laugerie } \\
\text { Haute }\end{array}$} & \multicolumn{2}{|c|}{ Aurignacien } & \multicolumn{2}{|c|}{$\underline{\text { Gravettien final }}$} & \multicolumn{2}{|c|}{ Total } \\
\hline & & & & & & No. & $\%$ & No. & $\%$ & No. & $\%$ \\
\hline amphibolite & 1 & - & - & - & - & 1 & 0,6 & - & - & 1 & 0,4 \\
\hline basalte & - & 2 & - & - & - & 2 & 1,3 & - & - & 2 & 0,7 \\
\hline calcaire encaissant & 1 & - & - & 1 & - & 1 & 0,6 & 1 & 0,8 & 2 & 0,7 \\
\hline calcaire lithographique jurassique & - & 4 & - & 2 & 2 & 4 & 2,6 & 4 & 3,3 & 8 & 2,9 \\
\hline cornéenne & - & - & - & 1 & - & - & - & 1 & 0,8 & 1 & 0,4 \\
\hline diorite & 1 & 1 & 1 & 2 & 3 & 3 & 1,9 & 5 & 4,2 & 8 & 2,9 \\
\hline gneiss & 12 & 5 & 2 & 1 & 2 & 19 & 12,3 & 3 & 2,5 & 22 & 8,0 \\
\hline granite & 2 & 1 & - & 3 & 4 & 3 & 1,9 & 7 & 5,8 & 10 & 3,6 \\
\hline grès & - & - & 1 & - & - & 1 & 0,6 & - & - & 1 & 0,4 \\
\hline quartz & 43 & 34 & 2 & 31 & 20 & 79 & 51,0 & 51 & 42,5 & 130 & 47,3 \\
\hline quartzite & 8 & 3 & - & - & 3 & 11 & 7,1 & 3 & 2,5 & 14 & 5,1 \\
\hline roche magmatique indéterminée & 1 & - & - & 5 & - & 1 & 0,6 & 5 & 4,2 & 6 & 2,2 \\
\hline roche siliceuse volcanique & - & - & - & 2 & - & - & - & 2 & 1,7 & 2 & 0,7 \\
\hline schiste & 3 & 1 & - & - & - & 4 & 2,6 & - & - & 4 & 1,5 \\
\hline silex & 1 & 21 & 4 & 26 & 10 & 26 & 16,8 & 36 & 30,0 & 62 & 22,5 \\
\hline trachyte & - & - & - & 1 & 1 & - & - & 2 & 1,7 & 2 & 0,7 \\
\hline Total & 73 & 72 & 10 & 75 & 45 & 155 & 100,0 & 120 & 100,0 & 275 & 100,0 \\
\hline
\end{tabular}

\section{RÉSULTATS}

\section{LES MATÉRIAUX EMPLOYÉS}

Différents types de matériaux ont été utilisés pour les activités de percussion. Le quartz est le matériau le plus employé sur les cinq sites étudiés ( $\mathrm{N}=130$, soit $47,3 \%$ des pièces), devant le silex $(\mathrm{N}=62$, soit $22,5 \%)$. Des matériaux tels que le gneiss, le quartzite ou le granite sont assez fréquents. D'autres matériaux tels que le basalte, le calcaire, la diorite, etc. sont présents, mais sont plutôt rares (Tableau 3).

Des différences de fréquence assez nettes apparaissent en fonction des sites ou des cultures (Tableau 3). L'emploi du quartz et du quartzite est encore plus dominant dans les sites aurignaciens (respectivement $51 \%$ et 7,1\%) que dans les sites du Gravettien final (42,5\% et 2,5\%). Le gneiss est quant à lui bien représenté dans les sites aurignaciens (12,3\%), mais il se révèle peu présent dans les sites du Gravettien final $(2,5 \%)$. Les objets en silex sont nettement moins nombreux dans les sites aurignaciens étudiés, mais il est probable que si l'ensemble des industries en silex des abris Blanchard et
Cellier avaient pu être examinées, ce nombre aurait assez fortement augmenté.

Des différences très nettes apparaissent également en fonction des types d'objets, attestant une sélection de certains matériaux pour des usages spécifiques. Ainsi, les percuteurs de concassage et les choppers sont quasi exclusivement en quartz ou en quartzite (Tableau 4), tandis que les maillets sont très fréquemment en calcaire lithographique ou en diorite, mais jamais en quartz. Par ailleurs, certains matériaux sont employés pour une fonction spécifique (par exemple le calcaire lithographique comme maillet ou le schiste comme enclume), alors que d'autres, tels que le quartz, le quartzite ou le gneiss, sont très ubiquistes.

Les sources et les modes d'acquisition de ces différents matériaux seront discutés plus bas.

\section{Les percuteurs classiques ou percuteurs de taille}

Sur les sites étudiés, les percuteurs utilisés pour le débitage des blocs de silex sont de loin les plus nombreux avec 82 pièces sur les 275 analysées. Cette catégorie de percuteurs est celle 


\begin{tabular}{|c|c|c|c|c|c|c|c|c|c|c|}
\hline Matière & 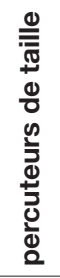 & 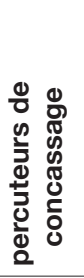 & 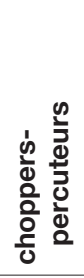 & 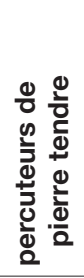 & 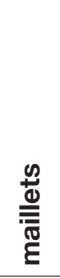 & 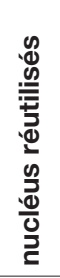 & 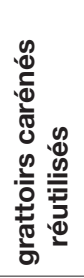 & 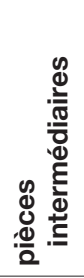 & 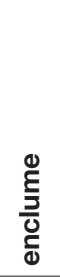 & 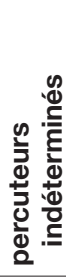 \\
\hline amphibolite & - & - & - & - & - & - & - & - & 1 & - \\
\hline basalte & - & - & - & - & - & - & - & - & 1 & 1 \\
\hline calcaire encaissant & - & - & - & 2 & - & - & - & - & - & - \\
\hline calcaire lithographique jurassique & - & - & - & - & 8 & - & - & - & - & - \\
\hline cornéenne & - & - & - & - & 1 & - & - & - & - & - \\
\hline diorite & - & - & 1 & - & 6 & - & - & - & 1 & - \\
\hline gneiss & 9 & 2 & 1 & - & 1 & - & - & - & 3 & 6 \\
\hline granite & 8 & 1 & - & - & - & - & - & - & 1 & 1 \\
\hline grès & - & - & - & - & - & - & - & - & 1 & - \\
\hline quartz & 56 & 35 & 18 & _- & - & - & - & _- & 11 & 20 \\
\hline quartzite & 6 & 5 & 2 & - & 1 & - & - & - & 2 & 5 \\
\hline roche magmatique indéterminée & 1 & - & - & - & - & - & - & - & - & - \\
\hline roche siliceuse volcanique & - & - & - & - & 2 & - & - & - & - & - \\
\hline schiste & - & - & - & - & - & - & - & - & 4 & - \\
\hline silex & 2 & - & - & - & - & 42 & 7 & 9 & - & 1 \\
\hline trachyte & - & - & - & - & 1 & - & - & - & - & 1 \\
\hline Total & 82 & 43 & 22 & 3 & 20 & 42 & 7 & 9 & 25 & 35 \\
\hline
\end{tabular}

TABLEAU 5. - Matériaux employés pour les percuteurs de taille.

\begin{tabular}{|c|c|c|c|c|c|c|}
\hline \multirow[b]{2}{*}{ Matière } & \multicolumn{3}{|c|}{ Aurignacien } & \multicolumn{2}{|c|}{ Gravettien final } & \multirow[b]{2}{*}{ Total } \\
\hline & Blanchard & Castanet & Cellier & Pataud & Laugerie-Haute & \\
\hline gneiss & 3 & 4 & 1 & - & 1 & 9 \\
\hline granite & 2 & 1 & - & 1 & 4 & 8 \\
\hline quartz & 15 & 9 & - & 24 & 8 & 56 \\
\hline quartzite & 1 & 1 & - & 4 & - & 6 \\
\hline roche magmatique indéterminée & 1 & - & - & - & - & 1 \\
\hline silex & - & - & 1 & - & 1 & 2 \\
\hline Total & 22 & 15 & 2 & 29 & 14 & 82 \\
\hline
\end{tabular}

qui présente la plus grande variabilité en termes de dimensions et de matériaux employés.

Les pièces entières présentent le plus souvent une dimension maximale comprise entre 50 et $100 \mathrm{~mm}$ (Fig. 8A) et un poids situé entre 90 et $500 \mathrm{~g}$. Quelques rares pièces présentent un poids plus important, mais ces dernières portent généralement des traces de percussions limitées qui semblent liées à des usages ponctuels.

Si la plupart des percuteurs de taille sont en quartz, on note quand même une certaine diversité dans le choix de la matière première avec la présence assez importante de gneiss, de granite ou de quartzite. On relève également de rares cas d'utilisation de rognons de silex. L'emploi majoritaire du quartz et la variabilité susmentionnée sont attestés sur l'ensemble des sites étudiés, qu'ils soient aurignaciens ou gravettiens final (Tableau 5).

Parmi ces percuteurs classiques, une pièce en granite de Laugerie-Haute présente des traces de pigment rouge exclusivement sur et autour d'une plage de stigmates de percussions (Chiotti et al. 2018 : 164, fig. 91d). L'association étroite de ces deux types de traces permet ainsi d'envisager que l'outil a pu être utilisé non pour la taille du silex, mais plutôt pour la fragmentation et le broyage de blocs de colorant.

\section{Les percuteurs de concassage}

Les percuteurs de concassage sont représentés par 43 pièces. Ces outils sont présents sur les cinq sites, mais ils sont nettement plus nombreux $(\mathrm{N}=33)$ sur les sites aurignaciens (Tableau 2). Ils sont caractérisés par des plages de traces de percussions d'étendue et d'intensité très importantes, qui ont le plus souvent provoqué une perte de matière, modifiant ainsi la forme des surfaces utilisées des galets. Cela se traduit dans la majeure partie des cas par un aplatissement des extrémités de ces galets (Fig. 9).

Les galets utilisés comme percuteurs de concassage comptent parmi les galets les plus volumineux utilisés en percussion (Fig. 8A). Les pièces les plus complètes ont des dimensions maximales comprises entre 80 et $130 \mathrm{~mm}$ et un poids compris entre 200 et $900 \mathrm{~g}$. Une pièce de l'abri Castanet atteint même $1400 \mathrm{~g}$. 


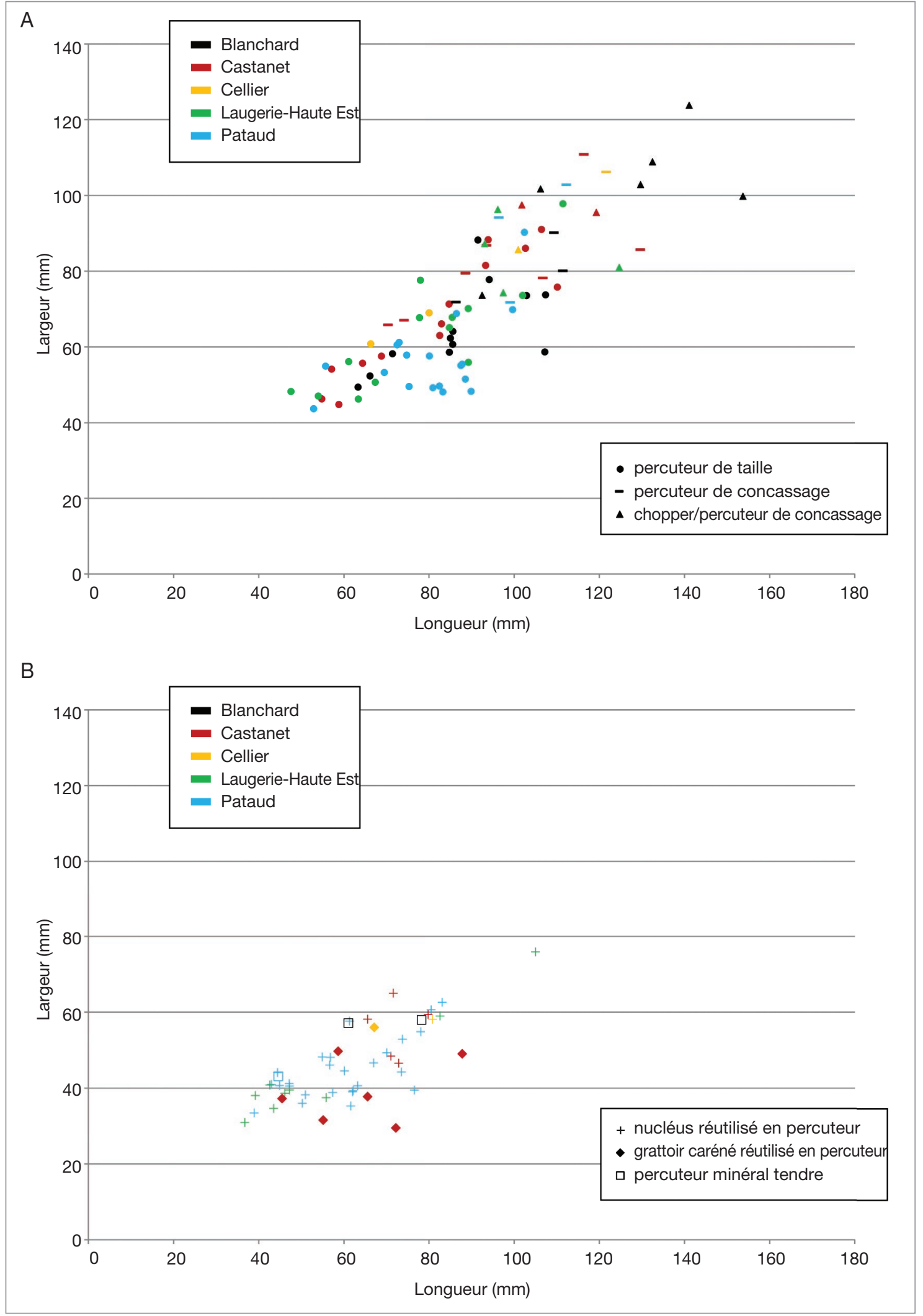

FIG. 8. - Dimensions (longueur-largeur en $\mathrm{mm}$ ) des différents types de percuteurs identifiés sur les cinq sites étudiés. 
A

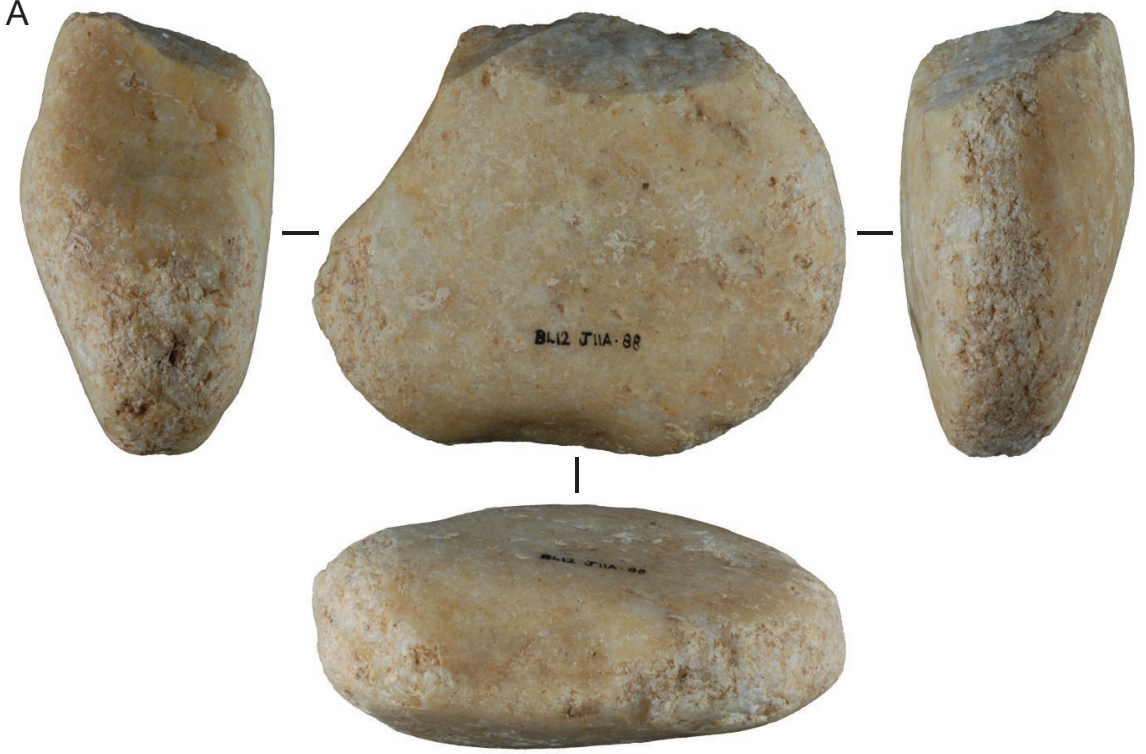

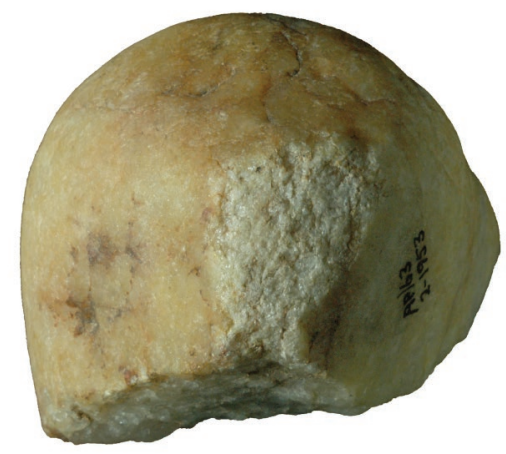

$\mathrm{B}$

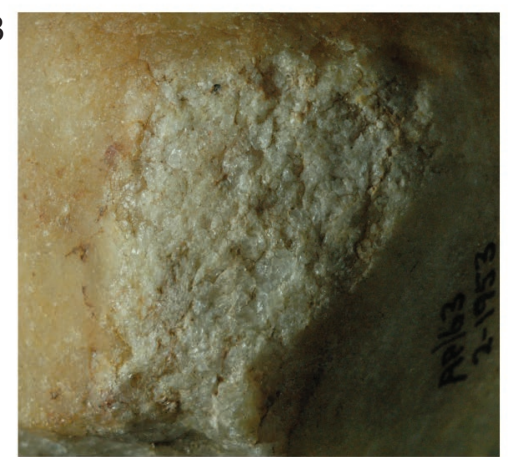

FIG. 9. - Percuteurs de concassage (clichés : L. Chiotti) : A, BL12-J11A-88, percuteur de concassage, abri Blanchard, fouille White, secteur 4/5; B, AP/63-21953, percuteur de concassage avec détail d'une plage de traces de percussions, abri Pataud, fouille Movius, couche 2 . Échelle : $5 \mathrm{~cm}$.

Les matériaux utilisés sont nettement moins diversifiés que dans la catégorie précédente : on décompte 35 pièces en quartz, cinq en quartzite, ainsi que deux pièces en gneiss à l'abri Blanchard et une en granite à l'abri Pataud.

Trois éclats de l'abri Blanchard (deux en quartz et un en quartzite) portant des traces de percussions relativement importantes sur leur talon proviennent probablement de tels percuteurs.

\section{Les choppers-percuteurs}

Tous les sites ont livré des galets présentant des détachements d'éclats volontaires dans le but de dégager des arêtes plus ou moins aiguës, et qui peuvent être rattachés à la catégorie typologique des choppers (Fig. 10).

Ces objets portent tous de très importantes traces de percussions et d'écrasement sur les arêtes dégagées par les détachements d'éclats. Il s'agit manifestement d'une volonté de dégager une arête destinée à être utilisée comme percuteur, probablement pour avoir une plus grande précision de la percussion. Les traces visibles sur ces arêtes sont très intenses, et très similaires à celles présentes sur les percuteurs de concassage (Fig. 10). La plupart de ces choppers présentent également des plages de traces de percussions très intenses sur les parties non taillées des galets, identiques à celles des percuteurs de concassage.

Les dimensions (de 90 à $150 \mathrm{~mm}$ de dimension maximale) et les poids (entre 400 et $1500 \mathrm{~g}$ ) de ces choppers-percuteurs sont également proches, voire même supérieurs à ceux des percuteurs de concassage (Fig. 8A).
Le matériau utilisé pour ces outils est presque exclusivement le quartz. Sur les 22 pièces identifiées, seules quatre sont en matériaux différents : une en diorite à l'abri Castanet, une en gneiss et deux en quartzite à l'abri Blanchard.

Comme pour les percuteurs de concassage, c'est dans les sites aurignaciens, et en particulier à l'abri Blanchard $(\mathrm{N}=11)$, que les choppers-percuteurs sont les plus nombreux $(\mathrm{N}=16$ sur un total de 22).

Quatre éclats de quartz (trois provenant de l'abri Blanchard et un issu de l'abri Castanet) portant de très importantes traces de percussions correspondent probablement à des éclats de ravivage des arêtes de ces choppers.

L'un des choppers en quartz de Laugerie-Haute porte une importante plage de traces de percussions sur l'extrémité opposée aux enlèvements de chopper. Cette plage est très fortement imprégnée de pigment rouge (Chiotti et al. 2018 : 68, fig. 33c). La distribution de ce pigment étant une nouvelle fois étroitement liée à celle des traces d'impacts, il semble crédible que ce percuteur ait pu être utilisé pour fragmenter et broyer des blocs de colorant.

\section{Les percuteurs de pierre tendre}

Seuls trois percuteurs de pierre tendre ont été identifiés : deux dans l'Aurignacien de l'abri Blanchard et un dans le Gravettien final de l'abri Pataud. Il s'agit très probablement de percuteurs utilisés pour la taille du silex. Dans le Gravettien final de l'abri Pataud, cela est compatible avec les observations effectuées sur les industries lithiques, puisque de nombreuses lames de petit gabarit portent des stigmates 


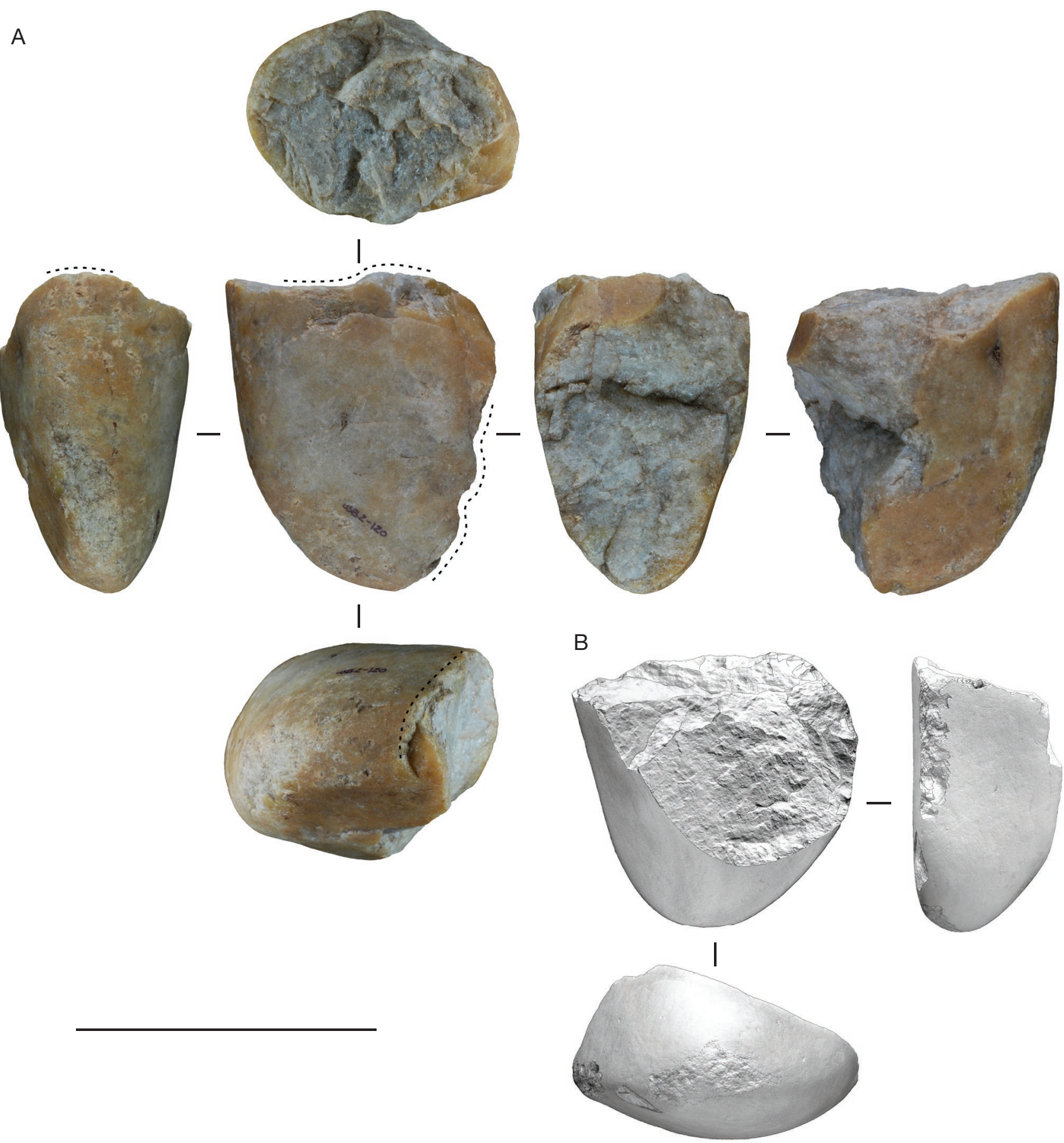

FIG. 10. - Choppers/percuteurs de concassage (clichés : L. Chiotti; dessin : J. Airvaux) : A, CL14-USB2-120, abri Cellier, déblais; B, L-H/352, Laugerie-Haute Est, fouilles Bordes, couche 38 . Échelle : $10 \mathrm{~cm}$.

de débitage au percuteur tendre (stigmates identifiés par Serge Maury). Dans l'Aurignacien de l'abri Blanchard, de tels stigmates n'ont pas été identifiés, mais seules les petites séries issues des fouilles récentes ont été analysées.

Deux sont en calcaire et proviennent du calcaire coniacien constituant l'encaissant des deux abris. Dans les deux cas, il s'agit d'objets de forme quasiment sphérique, constituées d'une multitude de petites facettes résultant de la percussion (Fig. 11A, B). L'exemplaire de l'abri Pataud figure parmi les percuteurs les plus petits $(45 \times 43 \times 40 \mathrm{~mm}$ pour un poids de $87 \mathrm{~g})$. Celui de l'abri Blanchard est un peu plus volumineux, mais ses dimensions $(61 \times 57 \times 48 \mathrm{~mm}$ pour un poids de $163 \mathrm{~g}$ ) ne détonnent pas par rapport à celles des percuteurs de taille en matériaux plus durs (Fig. 8A, B).

Au vu de la morphologie et de l'état d'usure de ces pièces - des traces de percussions sont présentes sur toute leur surface -, il est probable qu'ils aient été utilisés sur une longue durée. 
A

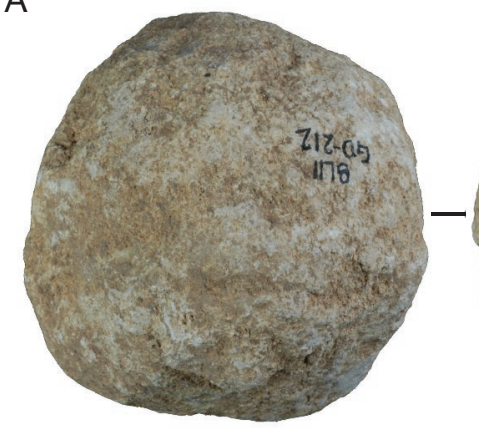

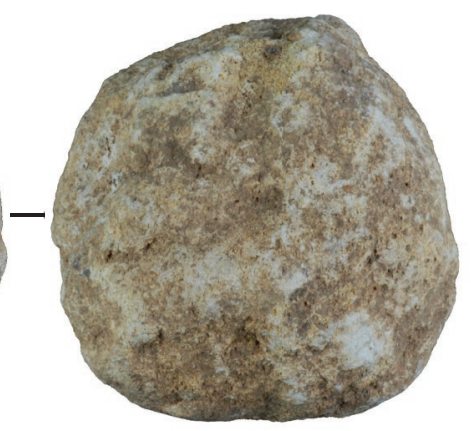

B

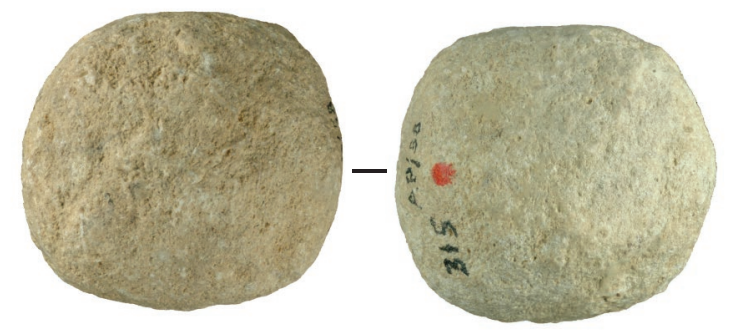

C
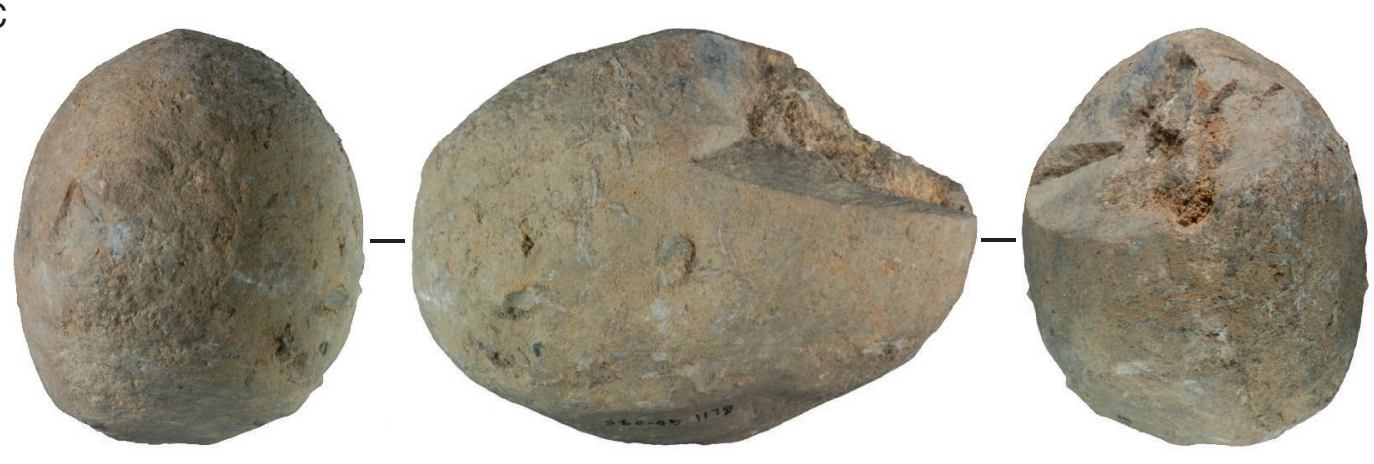

$\mid$

FIG. 11. - Percuteurs de pierre tendre (clichés : L. Chiotti) : A, BL11-GD-212, percuteur en calcaire coniacien encaissant, abri Blanchard, déblais; B, AP/58-2315, percuteur en calcaire coniacien encaissant, abri Pataud, fouille Movius, couche 2; C, BL11-GD-75, rognon de silex sénonien utilisé comme percuteur de pierre tendre, abri Blanchard, déblais. Échelle : $5 \mathrm{~cm}$.

La troisième pièce identifiée comme un percuteur minéral tendre est totalement différente. Il s'agit d'un petit nodule de silex sénonien local mal silicifié, ovalaire, très régulier, et qui porte des traces de percussions sur ses deux extrémités (Fig. 11C). Le cortex très épais et assez tendre de ce bloc nous incite à considérer cet objet comme un percuteur de pierre tendre, ce qui cohérent avec la définition que donne J. Pelegrin (Pelegrin 2000) pour ces objets (voir plus haut).

Sur l'une des deux extrémités, les traces de percussions assez développées, sont associées à un aplanissement et au détachement d'une petite esquille centimétrique. Sur l'autre extrémité, les traces de percussions sont moins nombreuses, mais on note le détachement accidentel d'au moins deux grands éclats. Ces éclatements sont également cohérents avec la plus faible durée d'emploi de ce matériau dont parle J. Pelegrin (Pelegrin 2000). L'un des bords allongés du nodule porte également quelques traces de percussions. Avec ses dimensions un peu plus importantes que celles des deux pièces précédentes $(78 \times 58 \times 48 \mathrm{~mm}$ pour un poids de $215 \mathrm{~g})$, cet objet entre également dans la variabilité dimensionnelle des autres percuteurs de taille (Fig. 8A, B).

\section{Les maillets}

À l'exception de l'abri Blanchard, les niveaux aurignaciens et gravettiens final étudiés ont tous livré des galets relativement petits, généralement allongés et qui portent sur leurs surfaces diverses traces d'utilisation (Fig. 12). Parmi eux, vingt petits galets portent, entre autres stigmates, des traces d'impacts regroupées sous la forme de plages plus ou moins denses, allant parfois jusqu'à créer une dépression.

Ces stigmates, ainsi que le gabarit des galets, permettent de classer ces objets dans la catégorie des maillets définie par S. de Beaune (voir plus haut).

Malgré des dimensions relativement variables, les galets sélectionnés ont globalement des formes très similaires : ils sont le plus souvent allongés et aplatis. Les longueurs varient entre 60 et $110 \mathrm{~mm}$ (à l'exception d'une pièce dont la longueur atteint $144 \mathrm{~mm}$ ) et les largeurs entre 20 et $70 \mathrm{~mm}$, ce qui représente des taux d'allongement (longueur/largeur) compris entre 1,5 et 4,1 (Fig. 13). Ces galets sont généralement très plats; leur épaisseur varie entre 8 et $32 \mathrm{~mm}$ et leur taux d'aplatissement (largeur/épaisseur) entre 1,2 et 4,1 (Fig. 14). À l'exception d'une pièce particulièrement volumineuse et massive (305 g) provenant de l'abri Pataud (Chiotti et al. 2018 : 103), ces objets sont légers (entre 20 et $130 \mathrm{~g}$ ).

Les matériaux employés sont variés, mais il s'agit généralement de roches à grain fin, assez compactes. On rencontre notamment des calcaires à grains fins (de type calcaire lithographique) et divers types de roches magmatiques, volcaniques ou métamorphiques (Tableau 6). Le quartz n’est jamais employé.

Les galets sélectionnés pour ces outils ne sont jamais transformés et ont fait l'objet d'une très forte sélection, avec le choix de matières premières particulières et de morphologies très régulières, alors que de tels galets sont rares dans la nature. 
A
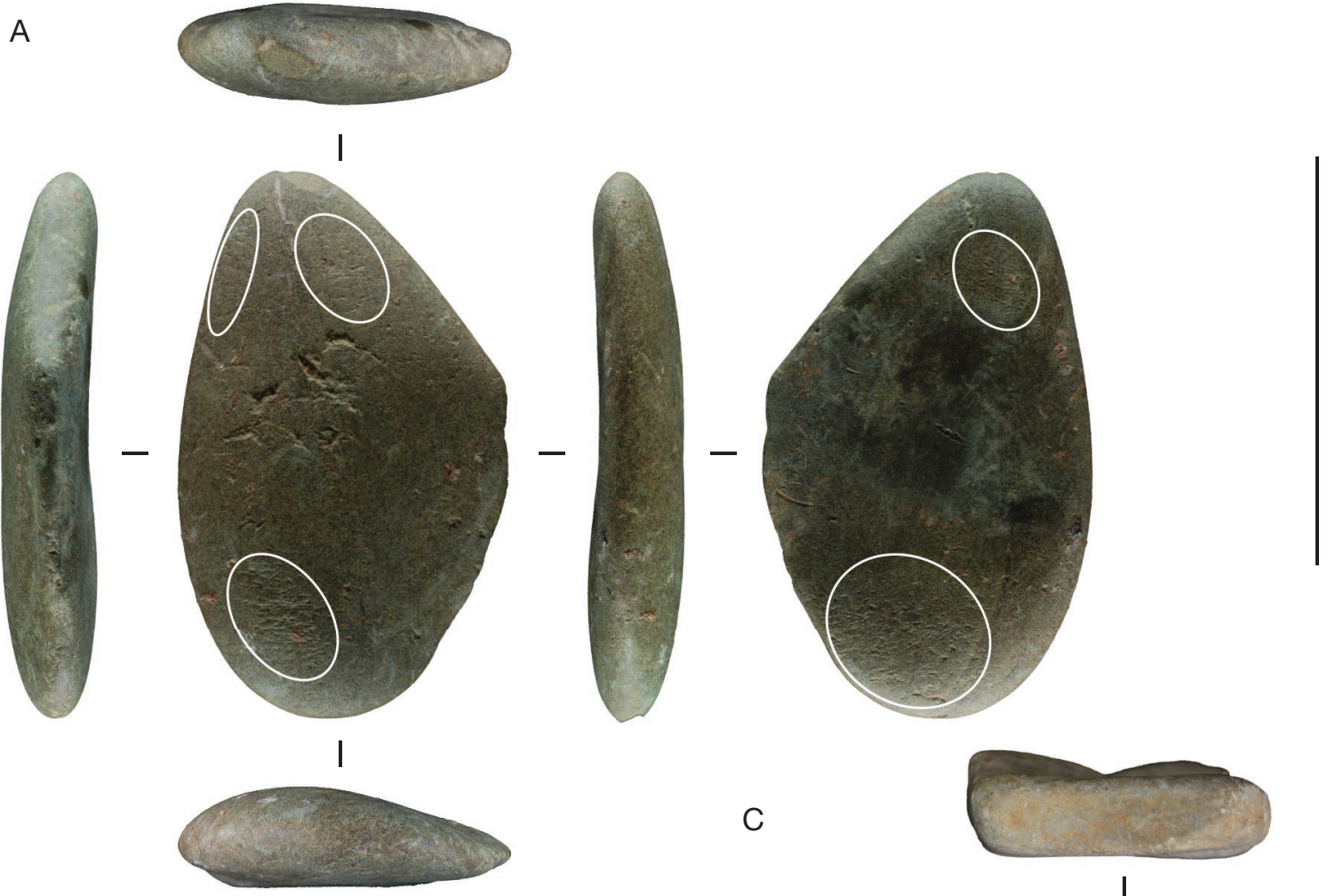

C
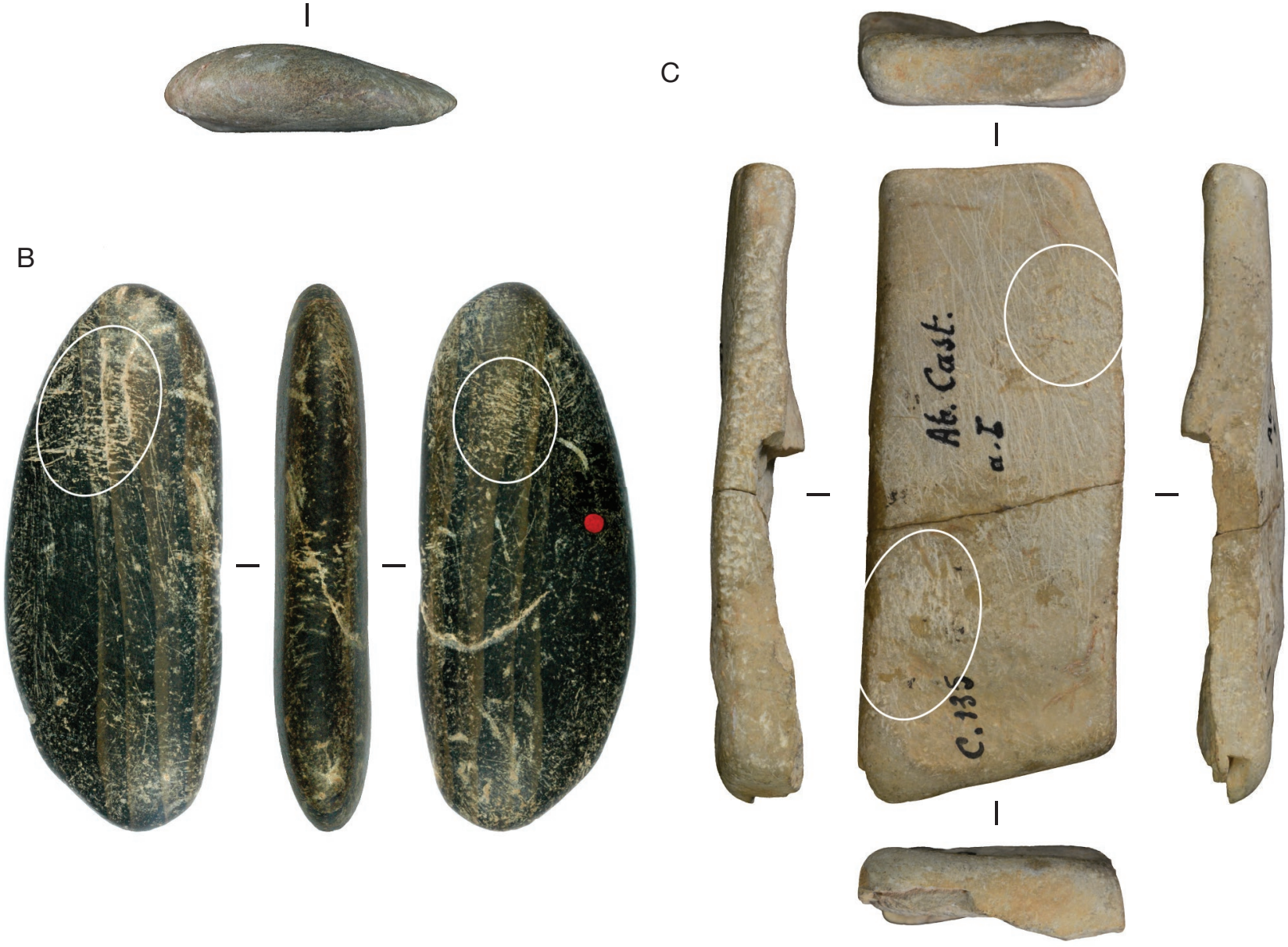

FIG. 12. - Maillets (clichés : L. Chiotti) : A, CL14-P29B-1, maillet en diorite, abri Cellier, fouille White; B, AP/58-2-291, maillet en roche siliceuse volcanique indéterminée, abri Pataud, fouille Movius, couche 2; C, CT-PEYR-33, maillet en calcaire lithostratigraphique jurassique, abri Castanet, fouille Peyrony. Échelle : 5 cm. 


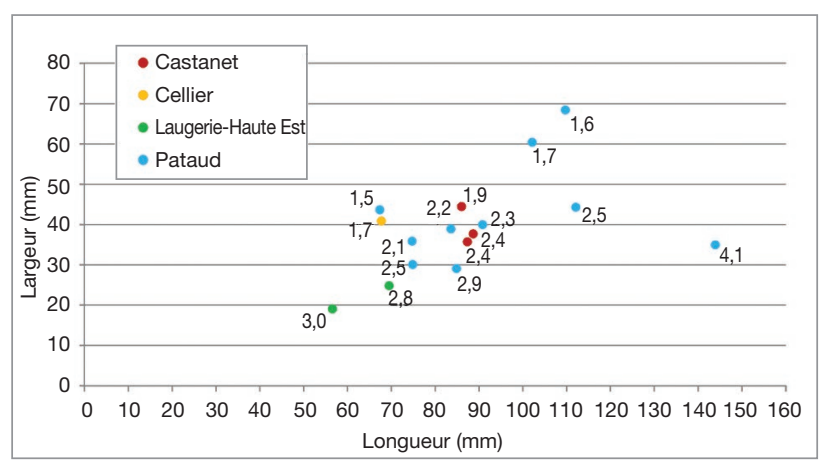

FIG. 13. - Dimensions (longueur-largeur en mm) des galets utilisés comme maillets et taux d'allongement des supports sélectionnés. Seules les pièces entières sont prises en compte.

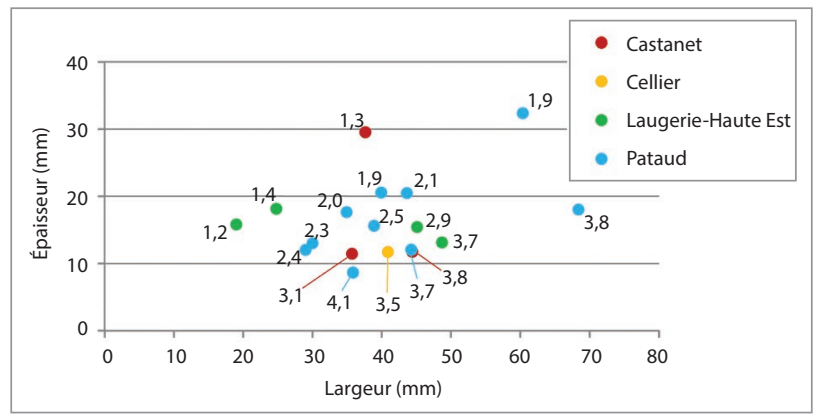

FIG. 14. - Dimensions (largeur-épaisseur en mm) des galets utilisés comme maillets et taux d'aplatissement des supports sélectionnés. Seules les pièces dont la section est complète sont prises en compte.

Bien qu'ils y soient peu fréquents la plupart des matériaux employés (gneiss, diorite, quartzite à chlorite) sont présents dans les alluvions de la Vézère et ont donc pu être collectés localement. La recherche de ces galets très particuliers a certainement dû impliquer un investissement en termes de temps. D'autre part, il s'agit d'objets aux fonctions multiples qui ont pu être utilisés sur de longues durées. À l'abri Pataud, certains d'entre eux ont pu également avoir un rôle symbolique : celui de mobilier funéraire. En effet, dans la couche 2 de l'abri Pataud une série d'objets (objets d'art et de parure, outils et vestiges fauniques) très particuliers ont été mis au jour. De par leur rareté, leur localisation le plus souvent à proximité des vestiges humains de ce niveau, leurs matières premières rares et/ou leur investissement technique, au moins une partie d'entre eux est considérée comme du mobilier funéraire (Chiotti et al. 2009, 2014). Certains des petits galets à traces d'utilisation multiples et diversifiées font partie de cette série d'objets.

\section{Les pièces en silex réutilisées en percussion}

Des pièces en silex portant des traces de percussions, parfois très importantes, sont présentes sur tous les sites, à l'exception de l'abri Blanchard. Pour ce dernier, l'absence de ce type d'objet pourrait résulter d'un biais lié à la non intégration des collections en silex issues des fouilles anciennes dans notre corpus d'étude.
Dans la plupart des cas, il s'agit de nucléus $(\mathrm{N}=41)$, beaucoup plus rarement de gros éclats $(\mathrm{N}=1)$. Les nucléus réutilisés sont des nucléus à éclats ou des nucléus laminaires prismatiques généralement parvenus à exhaustion. Sur les sites aurignaciens, il peut également s'agir de grattoirs carénés $(\mathrm{N}=6)$ ou d'ébauches de grattoirs carénés (Chiotti \& Cretin 2011; $\mathrm{N}=1$ ).

Certains de ces objets ont été si intensément utilisés en percussion que les traces résultant de cette action ont presque entièrement effacé les négatifs de débitage, jusqu’à conférer aux nucléus un aspect quasi sphérique. D’autres exemplaires présentent des stigmates de percussion plus limités et concentrés en une zone bien précise, suggérant ainsi un usage beaucoup moins intensif. Dans le cas des nucléus prismatiques laminaires, c'est souvent la base du nucléus qui a été percutée (qu'elle soit corticale ou non), alors que le plan de frappe est généralement peu ou pas utilisé. Le choix préférentiel de la base de ces nucléus comme partie percutante est sans doute lié à la forme de ces objets. La base, généralement plus arrondie semble en effet plus propice à un usage comme percuteur que le plan de frappe qui présente une surface plus plane et des arêtes plus saillantes.

Dans le cas des grattoirs carénés, la partie percutée peut être aussi bien le front de grattoir que l'extrémité opposée, mais il arrive aussi que tout le pourtour de l'objet soit utilisé ainsi qu'en témoigne l'exemplaire provenant de l'abri Cellier (Fig. 15A).

En plus des traces de percussions, certaines pièces portent des traces de poli ou de lustre, parfois très intenses, sur les parties non percutées (arêtes ou surfaces).

L'ensemble des stigmates observé sur ces objets semble donc attester un usage prolongé de certains d'entre eux.

À quelques exceptions près, les pièces en silex réutilisées comme percuteurs sont de dimensions modestes : il s'agit en effet des objets les plus petits et les plus légers identifiés parmi les différents types de percuteurs reconnus (Fig. 8B). Leurs longueurs sont comprises entre 30 et $80 \mathrm{~mm}$, leurs largeurs entre 30 et $60 \mathrm{~mm}$ et leurs poids entre 30 et $300 \mathrm{~g}$. Les pièces les plus petites sont souvent celles qui présentent les traces de percussions les plus intensives. Cependant ce n'est pas l'usage en percussion qui a conduit à une réduction de la taille de ces objets, mais il s'agit bien d'un choix de petits objets pour la percussion.

Bien que quelques spécimens soient présents dans les sites aurignaciens, cette catégorie de percuteurs est surtout attestée dans les deux sites du Gravettien final, et plus particulièrement à l'abri Pataud qui a livré plus de la moitié des pièces identifiées. Dans les sites aurignaciens, la moitié des nucléus choisis sont des grattoirs carénés.

\section{Les pièces intermédiaires}

L'abri Castanet a livré neuf pièces volumineuses en silex portant des traces de percussions et d'écrasement, relativement peu intenses dans la plupart des cas, sur des parties opposées des objets (Fig. 16). Parmi les trois sites aurignaciens étudiés, ce type d'objet n'a été identifié que dans les collections de l'abri Castanet. Cependant, dans la mesure où nous n'avons pas pu étudier l'intégralité de l'industrie en silex des deux autres sites, nous ne pouvons pas exclure la possibilité qu'y figurent des objets similaires. 
A
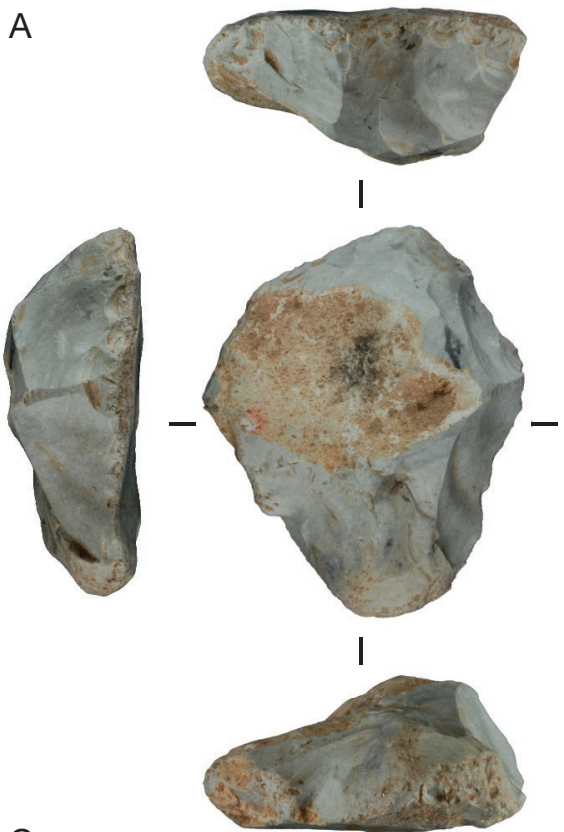

C
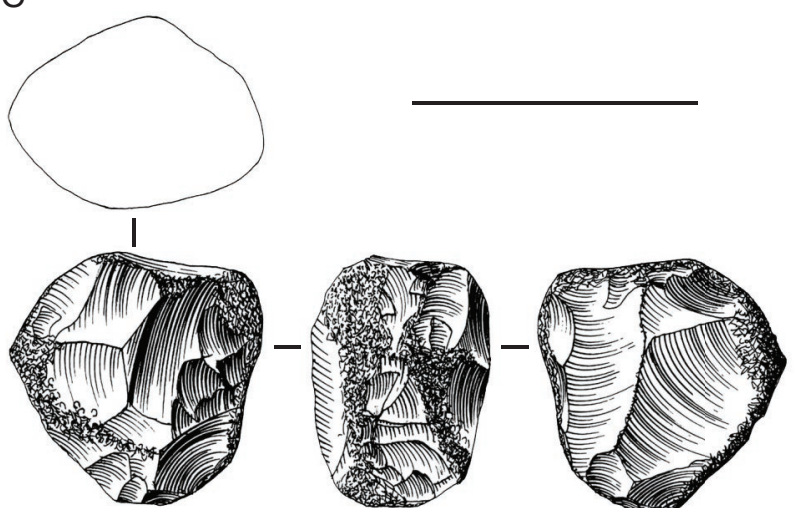
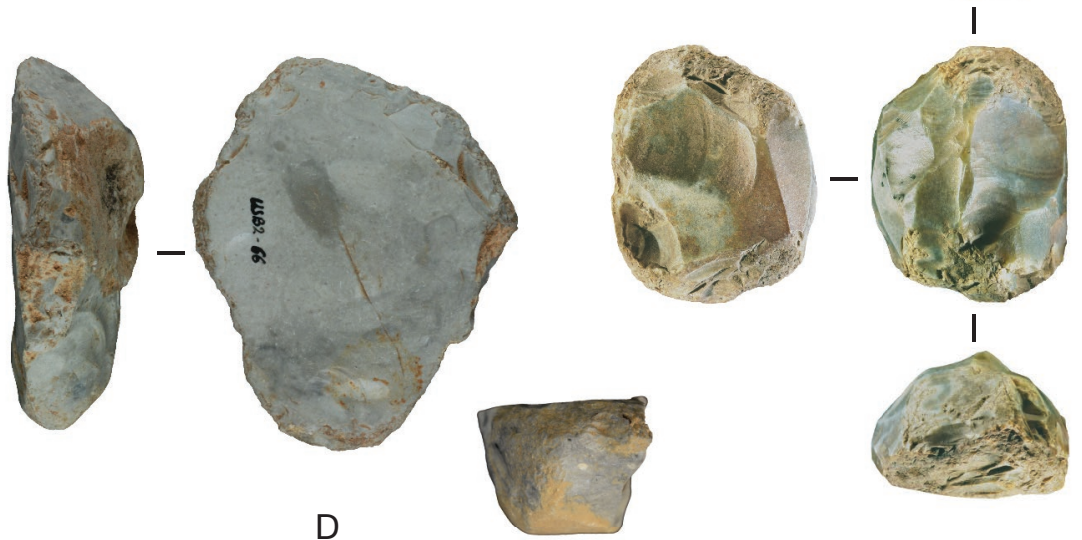

I

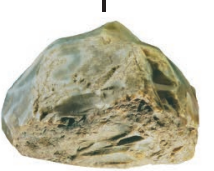

D
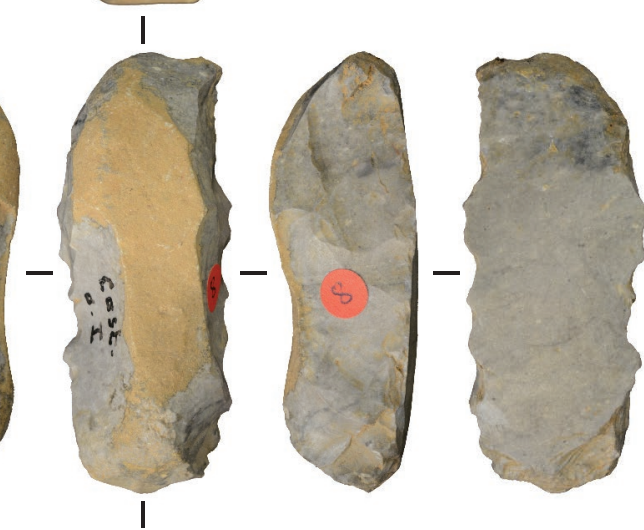

FIG. 15 - Pièces en silex réutilisées en percussion : A CL14-USB2-66, grattoir caréné, abri Cellier, déblais (clichés : L Chiotti); $\mathbf{B}$, L-H/LIII, nucléus, avec de forts polis sur les arêtes et les surfaces, Laugerie-Haute Est, fouille Peyrony, couche F (clichés : Ph. Jugie, MNP); C, AP/58-2-2904, nucléus, abri Pataud, fouille Movius, couche 2 (dessin: M. Dauvois); D, CT-PEYR-54, ébauche de grattoir caréné, abri Castanet, fouille Peyrony (clichés : L. Chiotti). Échelle : 5 cm.

TABLEAU 6. - Matériaux employés pour les maillets.

\begin{tabular}{|c|c|c|c|c|c|c|}
\hline \multirow[b]{2}{*}{ Matière } & \multicolumn{3}{|c|}{ Aurignacien } & \multicolumn{2}{|c|}{ Gravettien final } & \multirow[b]{2}{*}{ Total } \\
\hline & Blanchard & Castanet & Cellier & Pataud & Laugerie-Haute & \\
\hline calcaire lithographique jurassique & - & 4 & - & 2 & 2 & 8 \\
\hline cornéenne & - & - & - & 1 & - & 1 \\
\hline diorite & _- & _- & 1 & 2 & 3 & 6 \\
\hline gneiss & - & - & - & 1 & - & 1 \\
\hline quartzite & - & - & - & 1 & - & 1 \\
\hline roche magmatique indéterminée & - & _- & _- & 2 & _- & 2 \\
\hline trachyte & - & - & - & 1 & - & 1 \\
\hline Total & 0 & 4 & 1 & 10 & 5 & 20 \\
\hline
\end{tabular}

Les supports utilisés sont très diversifiés et attestent d'un réemploi, tout comme la catégorie précédente. Ainsi, ont été choisis : un grattoir caréné, une ébauche de grattoir caréné, deux nucléus laminaires prismatiques, trois nucléus à éclats et deux gros éclats bruts. Ces supports ont pour points communs un aspect généralement massif et des dimensions maximales assez importantes comprises entre 60 et $100 \mathrm{~mm}$. Leur poids est quant à lui situé entre 90 et $400 \mathrm{~g}$. La diversité des sup- 
A
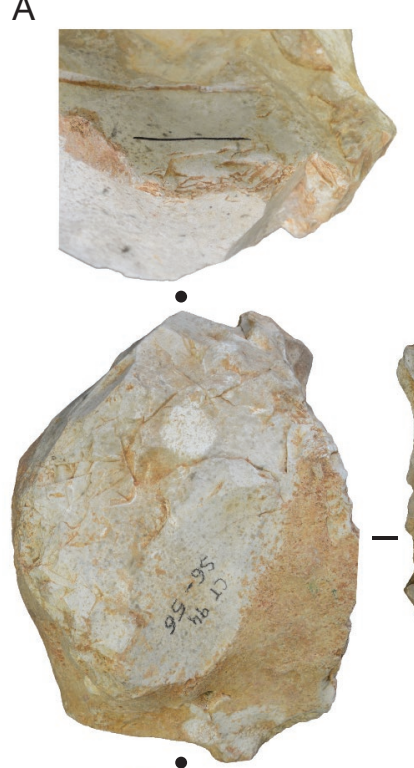

$\bullet$

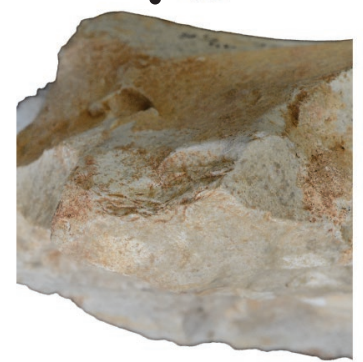

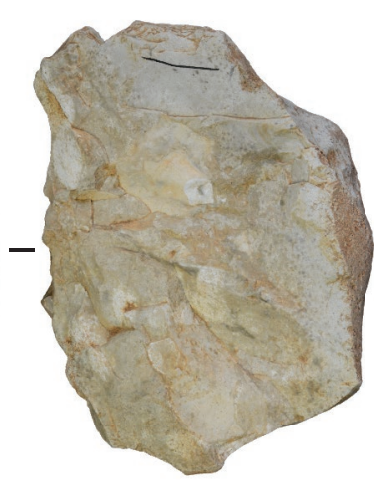

$\mathrm{D}$
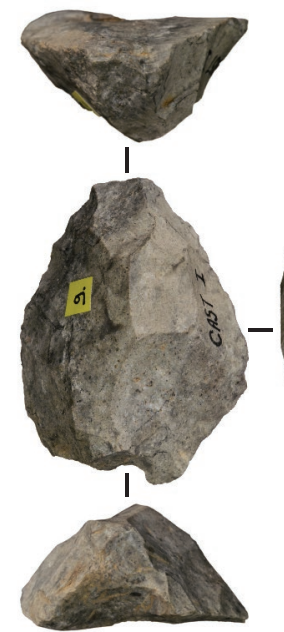
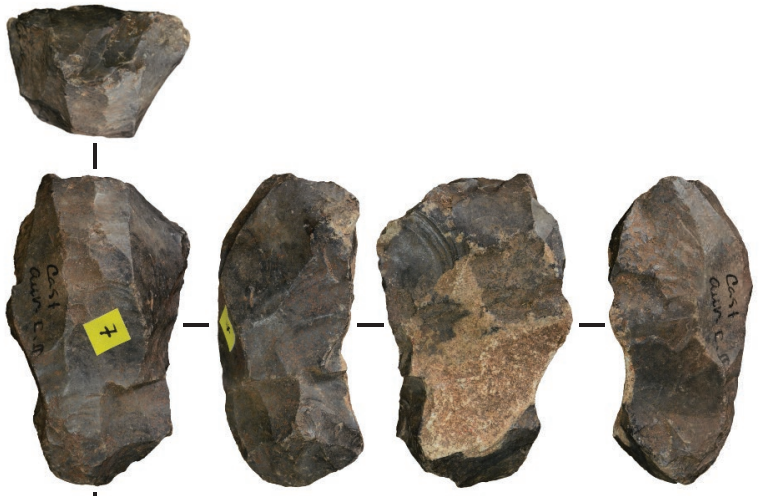

C
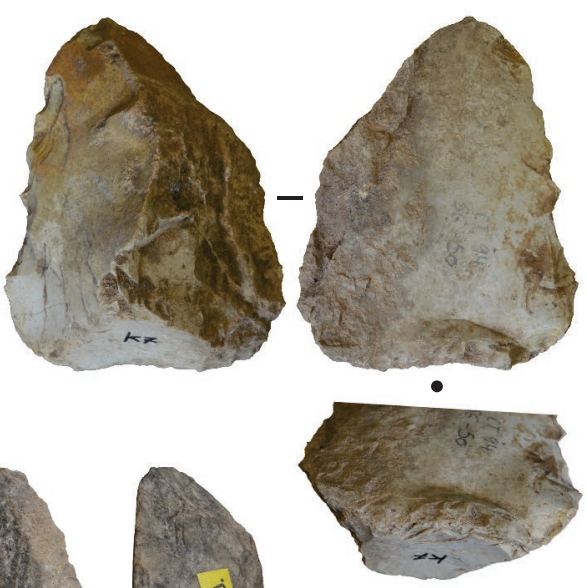

FIG. 16. - Objets en silex de l'abri Castanet réutilisés comme pièces intermédiaires (clichés : L. Chiotti) : A, CT94-S6-56, nucléus à éclats irrégulier; B, CT-PEYR-49, nucléus laminaire prismatique; C, CT94-S6-50, gros éclat brut épais; D, CT-PEYR-50, grattoir caréné. Échelle : $5 \mathrm{~cm}$.

ports, ainsi que la faible intensité des traces de percussions permettent d'envisager un choix de supports assez opportuniste pour des usages plutôt ponctuels.

La nature des traces observées ainsi que leur position sur les supports (localisation sur deux pôles opposés des objets) laisse supposer un usage comme pièce intermédiaire entre le percuteur et le matériau à travailler. La nature de ce matériau reste en l'état actuel inconnue.

\section{Les enclumes}

Les cinq sites étudiés ont livré un total de 25 pièces pouvant correspondre à la description d'une enclume. Toutefois, cellesci sont nettement plus nombreuses sur les sites aurignaciens $(\mathrm{N}=22)$ que sur les sites du Gravettiens final $(\mathrm{N}=3)$. Parmi elles, 13 pièces sont également décomptées dans des catégories décrites précédemment. Il s'agit de huit percuteurs de concassage (Fig. 17D), trois choppers-percuteurs de concassage
(Fig. 17C) et deux percuteurs de taille qui, tous, portent des stigmates d'utilisation comme enclume.

Parmi les douze autres enclumes recensées, plusieurs portent elles aussi des traces relatives à une autre fonction : trois enclumes portent des stries attestant un probable usage comme billot, une porte des traces de pigment en forme de "gouttes" et une autre a peut-être servi de meule. Quatre autres enclumes présentent sur tout ou partie de leur pourtour des négatifs d'enlèvements résultant probablement d'une mise en forme intentionnelle de l'objet (Fig. 17B).

Une telle multiplicité d'usages est très fréquente pour les enclumes paléolithiques. Ainsi S. de Beaune signale qu'elles «ont souvent également servi de billot, de meule et de palette comme l'indique la diversité de leurs traces (impacts, mais aussi stries, poli et restes de colorant)». Elle précise également que «les petites enclumes ont souvent aussi servi de percuteurs, comme le révèlent les nombreuses traces 
A
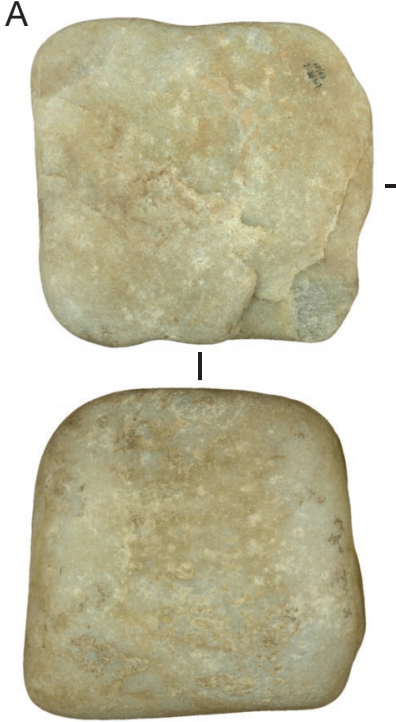

C

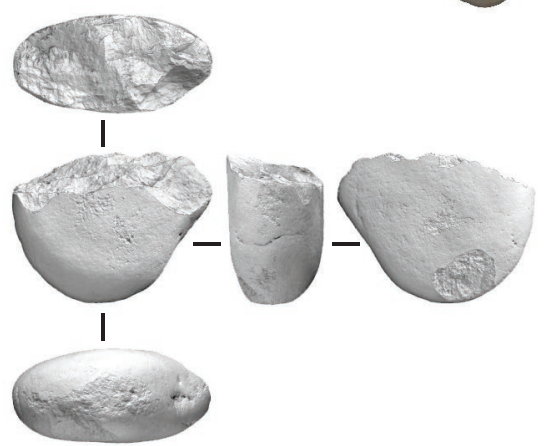

$\mathrm{D}$
B
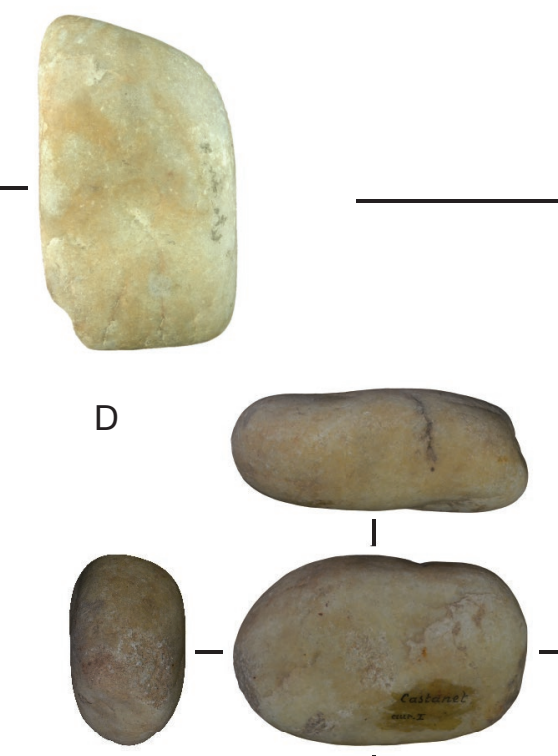

|
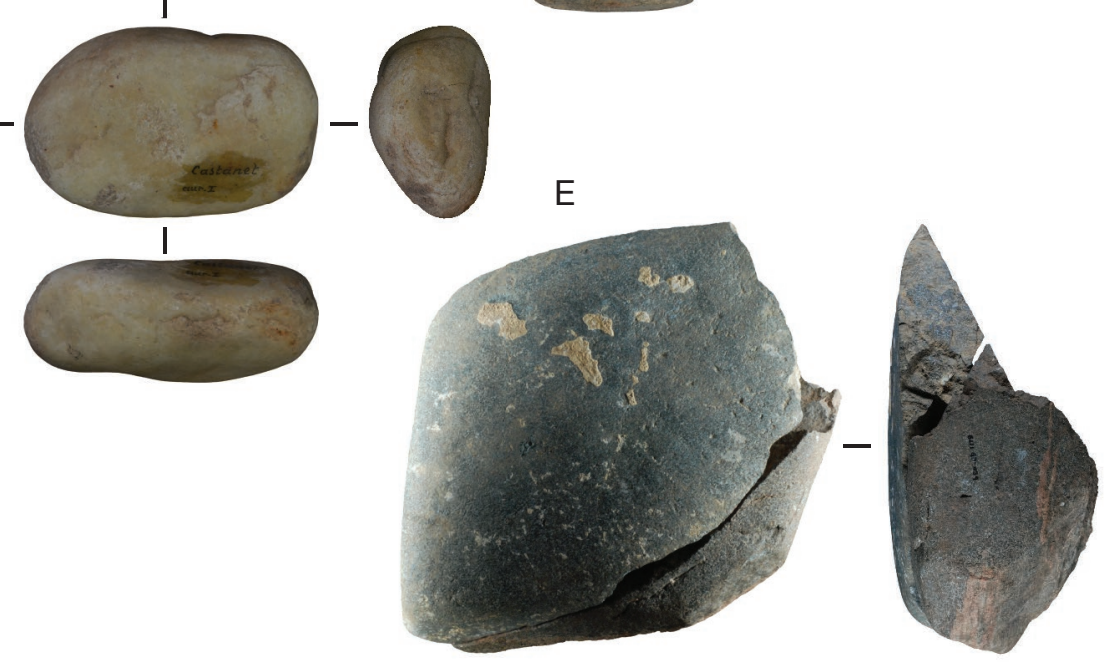

FIG. 17. - Enclumes (clichés : L. Chiotti; dessin : J. Airvaux) : A, AP/63-2-36907, enclume en quartz, abri Pataud, fouille Movius, couche 2; B, CT-PEYR-53, enclume en schiste vert dont le pourtour est façonné par des enlèvements d'éclats, abri Castanet, fouille Peyrony; C, L-H/56, chopper/percuteur de concassage/ enclume en quartz, Laugerie-Haute Est, fouille Peyrony, couche F; D, CT-PEYR-57, percuteur de concassage/enclume en quartz, abri Castanet, fouille Peyrony; E, BL11-GD-39 + BL11-GD-240, enclume en diorite, abri Blanchard, déblais des anciennes fouilles. Échelle : $10 \mathrm{~cm}$.

d'impacts visibles sur leurs extrémités et/ou leurs flancs» (Beaune $2000: 49)$.

Les dimensions des enclumes identifiées dans les sites étudiés sont extrêmement variables. Leurs dimensions maximales vont d'une dizaine de centimètres à plus de $37 \mathrm{~cm}$, tandis que leurs poids se situent entre $300 \mathrm{~g}$ et $12,4 \mathrm{~kg}$. On retrouve parmi ces objets les deux types d'enclumes paléolithiques décrits par S. de Beaune : «d'une part des blocs et des dalles pesant plusieurs kilos [...] avec une base bien stable, et d'autre part des galets de plus petites dimensions, de forme hexagonale, quadrangulaire ou ovale» (Beaune 2000 : 46).

Le quartz est le matériau le plus couramment employé $(\mathrm{N}=11)$, mais de nombreuses autres roches ont également été utilisées, en particulier des roches éruptives, magmatiques ou métamorphiques (Tableau 7).

À l'exception d'une grosse enclume quadrangulaire découverte à l'abri Pataud (Chiotti et al. 2018 : 54), toutes celles qui sont en quartz et en quartzite ont également été utilisées comme percuteurs. Parmi les pièces ayant assumé ces deux fonctions, une seule est constituée d'un autre matériau que le quartz : une pièce en granite de l'abri Pataud (Chiotti et al. 2018 : 55).
La présence de ces enclumes dans les différentes séries pose la question de leur utilisation. Nous avons vu plus haut qu'il est possible que certains percuteurs de concassage puissent correspondre à des outils destinés à la fragmentation des os. Or, lors de ses expérimentations sur la fracturation des os, G. Célérier a montré la nécessité de travailler sur un support dur (Célérier \& Kervazo 1988). Par conséquent, certaines enclumes pourraient avoir été utilisées comme support pour des activités de fragmentation des os. Néanmoins, bien d'autres utilisations sont envisageables (voir plus bas).

La répartition des différents types d'outils, montre que c'est dans les sites aurignaciens que les percuteurs de concassage $(\mathrm{N}=33$ sur 43 ) et les chopper-percuteurs de concassage ( $\mathrm{N}=16$ sur 22 ) sont les plus nombreux, en particulier à l'abri Blanchard, mais aussi à l'abri Castanet (Tableau 2). Or, il apparait que c'est également sur ces mêmes sites que les enclumes sont les mieux représentées $(\mathrm{N}=22$ sur 25 ; Tableau 2). Quelle que soit la, ou les matière(s) travaillée(s) à l'aide de ces outils, il est donc possible qu'il existe un lien fonctionnel entre les percuteurs de concassage et les enclumes. 
TABLEAU 7. - Matériaux employés pour les enclumes.

\begin{tabular}{|c|c|c|c|c|c|c|}
\hline \multirow[b]{2}{*}{ Matière } & \multicolumn{3}{|c|}{ Aurignacien } & \multicolumn{2}{|c|}{ Gravettien final } & \multirow[b]{2}{*}{ Total } \\
\hline & Blanchard & Castanet & Cellier & Pataud & Laugerie-Haute & \\
\hline amphibolite & 1 & - & - & - & - & 1 \\
\hline basalte & - & 1 & - & - & - & 1 \\
\hline diorite & 1 & - & - & - & - & 1 \\
\hline gneiss & 3 & - & - & - & - & 3 \\
\hline granite & - & - & - & 1 & - & 1 \\
\hline grès & - & - & 1 & - & - & 1 \\
\hline quartz & 3 & 6 & - & 1 & 1 & 11 \\
\hline quartzite & 2 & & - & - & - & 2 \\
\hline schiste & 3 & 1 & - & - & - & 4 \\
\hline Total & 13 & 8 & 1 & 2 & 1 & 25 \\
\hline
\end{tabular}

\section{DISCUSSION}

\section{DIVERSITÉ DES ACTIVITÉS}

\section{ATTESTÉES PAR LES MLHS PERCUTÉS}

Lors de ses études des MLHS, L. Daulny a classé les activités représentées par ces matériaux en trois fonctions principales (Daulny 2006a) : 1) une fonction de structuration de l'espace d'habitat et de l'espace mortuaire; 2) une fonction domestique qui peut se diviser en activités techniques et activités à but alimentaire; et 3) une fonction esthétique/artistique, rituelle et ludique que nous préférons qualifier de symbolique (Chiotti et al. 2018).

Les différents types d'outils rencontrés sur les cinq sites étudiés attestent d'activités de percussions variées qui correspondent quasiment toutes à des fonctions domestiques au sein de l'habitat. Au sein de ces dernières, la majeure partie des objets percutés étudiés ici entre dans le groupe des activités techniques.

C'est le cas des activités liées au débitage des blocs de silex qui sont majoritairement représentées avec la catégorie des percuteurs classiques (ou percuteurs de taille), ainsi qu'avec celle des percuteurs minéraux tendres. Bien que cela ne soit pas certain, il est également possible que les nucléus en silex percutés participent à ces activités de débitage du silex, au moins pour une partie d'entre eux.

Ces nucléus en silex percutés pourraient correspondre à deux autres types d'activités techniques : soit le travail de la pierre (hypothèse d'un usage comme bouchardes, notamment pour la préparation de surface de blocs calcaires), soit l'allumage de feux (hypothèse d'un usage comme briquets). Ces objets ne présentant pas tous des traces strictement identiques (tant en nature qu'en intensité), il est possible qu'ils aient participé à plusieurs de ces activités.

Pour les petits galets allongés présentant des plages de traces de percussions formant souvent des cupules sur les extrémités de leurs faces, $S$. de Beaune propose sur la base d'expérimentations et d'études de séries archéologiques (notamment celle de la grotte d'Isturitz, Saint-Martin-d'Arberoue, Pyrénées-Atlantiques) un usage comme maillet (Beaune 2000). Ces galets permettent d'effectuer un travail en percussion légère et précise sur une pièce intermédiaire qui étant donné les traces observées ne peut être qu'en matière dure telle que de la pierre. Selon les hypothèses de l'auteure, la pièce intermédiaire pourrait-être un objet en silex (burin, perçoir ou lame brute non retouchée), le tout étant utilisé "pour effectuer un travail précis sur une matière dure animale (os, bois de cervidé ou ivoire) et éventuellement sur des solides souples tels que la peau ou le cuir». (Beaune 2000 : 108). Ces objets seraient donc également destinés à des activités techniques telles que le façonnage matières dures d'origine animale ou la perforation de peaux.

Les activités de fracturation sont bien représentées au sein du corpus étudié avec d'une part les percuteurs de concassage et les choppers percutés et d'autre part les enclumes. Nous avons vu qu'il existe probablement un lien entre les percuteurs et choppers destinés aux activités de fracturation et les enclumes qui peuvent servir de support à ces dernières. En revanche le matériau travaillé est plus difficile à déterminer. Comme cela a déjà été évoqué plus haut, différents auteurs ont proposé un usage pour la fragmentation des os (HenriMartin 1910; Célérier \& Kervazo 1988; Beaune 1993a, 2000; Daulny 2006b; Pop et al. 2018; etc.).

Cependant, l'usage de ces objets pour la fragmentation de l'os n'est pas prouvé et/ou ne concerne probablement pas tous les objets en question. Un travail sur d'autres types de matériaux est parfois proposé, comme par exemple des noix (Beaune 2000; Pop et al. 2018), essentiellement sur la base de comparaisons ethnographiques. Seules des analyses tracéologiques et/ou des analyses de résidus pourraient éventuellement permettre de trancher sur ce sujet. Quoi qu'il en soit, les différentes hypothèses proposées rattachent plus ces objets des activités à but alimentaire (récupération de la moelle des os, fracturation de fruits à coque) qu’à des activités techniques.

Les enclumes peuvent aussi servir de support pour le débitage du silex par percussion bipolaire, encore appelée débitage sur enclume (Mourre \& Jarry 2009-2010), et donc participer également aux activités techniques. Cependant, ce type de débitage n'a été identifié sur aucun des cinq sites étudiés.

Aux pièces impliquées dans les activités de fracturation, il faut probablement rajouter les pièces intermédiaires qui prennent place entre le percuteur et le matériau à travailler pour une plus grande précision de la percussion, mais qui participent aux mêmes types d'activités. Là encore, le matériau travaillé n'est pas connu. 
Parmi le corpus étudié, quelques rares objets peuvent correspondre à des fonctions symboliques. Il s'agit d'objets du Gravettien final de l'abri Pataud, considérés comme probable mobilier funéraire (Chiotti et al. 2009, 2014). C'est en particulier le cas de deux galets à traces multiples (AP/58-2-430 et AP/63-2-1757) portant notamment les stigmates d'une utilisation comme maillet (Chiotti et al. 2018). Pour ces objets, la fonction symbolique est en fait une fonction secondaire. Il s'agit avant tout d'outils à fonction domestique qui, étant donné leurs stigmates d'utilisation nombreux et variés, ont probablement eu une très longue durée d'utilisation. La fonction symbolique n'intervient qu'à la fin de la «vie» de ces objets, lorsqu'ils ont probablement été déposés pour accompagner leur propriétaire dans la mort.

Deux autres objets percutés peuvent avoir un lien avec une fonction symbolique : le percuteur en granite et le chopper en quartz du Gravettien final de Laugerie-Haute sur lesquels ont été identifiées de traces de pigment rouge, et qui ont pu être utilisés pour la fragmentation et/ou le broyage de blocs de colorant. Ici encore il s'agit en premier lieu d'outils à fonction domestique (fracturation, broyage) mais pour la préparation d'un matériau à usage potentiellement symbolique (colorant). Néanmoins, un tel lien avec un usage symbolique reste hypothétique puisque les matières colorantes peuvent avoir de nombreux usages, certains domestiques (pouvoir abrasif pour le polissage, pouvoir siccatif, etc.) et d'autres symboliques (pouvoir colorant pour la réalisation de peintures, usage funéraire, etc.) (Salomon 2009).

\section{EXPLOITATION DES RESSOURCES MINÉRALES}

La quasi-totalité des matériaux employés (amphibolite, basalte, cornéenne, diorite, gneiss, granite, quartz, quartzite, schiste et trachyte) pour le matériel de percussion sur les cinq sites étudiés a été récolté sous la forme de galets de rivière. À l'exception du basalte et du trachyte, tous ces matériaux proviennent du Massif Central et sont drainés par la Vézère. Ils sont donc disponibles à proximité immédiate des abris, puisque la Vézère ou ses alluvions sont dans tous les cas à moins de 300 mètres des sites.

Les seuls matériaux collectés sous une autre forme que des galets sont le calcaire encaissant $(\mathrm{N}=2)$ qui est disponible directement dans les abris et le grès $(\mathrm{N}=1)$ qui est disponible sur les plateaux au-dessus des abris. Dans les cinq sites, l'exploitation des MLHS utilisés en percussion est donc essentiellement locale, voire ultra locale.

Quelques rares pièces témoignent d'une collecte un peu plus lointaine. Le basalte et le trachyte qui provienne également du Massif Central sont absents de la Vézère, mais ils sont drainés par la Dordogne. Ils sont donc disponibles à une distance minimale variant de 10 à $20 \mathrm{~km}$ en fonction des sites. Ces matériaux étant très rares $(\mathrm{N}=4)$, il est possible qu'ils aient fait l'objet d'une collecte occasionnelle lors des déplacements des groupes.

Les petits galets de calcaire lithographique jurassique $(\mathrm{N}=8)$ sont avérés dans les alluvions de la Dordogne, mais pas dans ceux de la Vézère [communication personnelle A. Morala, malgré ses très nombreuses prospections régionales, cet auteur n’a jamais retrouvé de galets similaires dans la Vézère (Chiotti et al. 2018)]. Cependant, cette rivière traverse les formations jurassiques du nord du Périgord, elle reste donc susceptible de fournir de tels galets.

Le cas des objets en silex utilisés lors d'activités de percussion est un peu particulier. Comme il s'agit de la réutilisation de pièces déjà taillées, et donc disponibles directement sur le site, leur origine peut être considérée comme ultra locale. Cependant si l'on considère le matériau en lui-même il y a de nettes différences entre les sites aurignaciens et ceux du Gravettien final. Dans les premiers, il s'agit toujours de silex sénoniens locaux, alors que dans les seconds on trouve des silex d'origine parfois beaucoup plus lointaine. Ceux de l'abri Pataud sont majoritairement en silex sénonien local, mais on en trouve également un en silex campanien du Bergeracois, un en silex turonien du Fumélois et un en silex exogène indéterminé (Chiotti et al. 2018). Quant à ceux de Laugerie-Haute ils sont majoritairement en silex exogènes : deux en silex campanien du Bergeracois, un en silex turonien du Fumélois, un en silex jaspoïde hettangien du Bassin de Brive et un en silex maestrichtien à Lepidorbitö̈des du sud du Bassin Aquitain; seuls trois sont en silex sénonien local (Chiotti et al. 2018). Le choix de ces matériaux exogène pose question, et il est possible que l'origine ou la qualité de ces matériaux ait joué un rôle dans leur choix pour les activités de percussion lors du Gravettien final.

Les données disponibles sur les industries en silex des cinq sites, et en particulier sur l'origine des matériaux employés, sont très disparates :

1) pour la couche 2 de l'abri Pataud l'ensemble du matériel a été étudié, dans le cadre d'une thèse de doctorat pour la collection Movius (Kong-Cho 1997) et de la publication des fouilles pour la collection 2005-2015 (Chiotti et al. 2013);

2) pour le Gravettien final de Laugerie-Haute Est, le matériel de la couche F de D. Peyrony et des couches 36 et 38 de F. Bordes a été analysé dans le cadre de la thèse d'État de P.-Y. Demars (1994), mais cette étude ne concernait que l'outillage retouché;

3) pour l'abri Castanet nous avons étudié le matériel de la fouille 2005-2010 dans le secteur sud (Chiotti et al. 2015); le matériel de la fouille 1994-1998 dans le secteur sud a été étudiée par J. Pelegrin (Pelegrin \& White 1998); le matériel de la fouille Peyrony (essentiellement de l'outillage retouché) a été analysé par P.-Y. Demars (1994);

4) pour l'abri Blanchard, seul le matériel des deux lambeaux de couches fouillés en 2011-2012 a été analysé (données personnelles de l'auteur non publiées);

5) pour l'abri Cellier, la partie de la collection Collie conservée au Musée national de Préhistoire (essentiellement de l'outillage retouché) a été étudiée par P.-Y. Demars (1994) et celle conservée au Beloit Museum par A. D. Woods (2011).

Malgré ces disparités d'analyse, on peut noter que sur les cinq sites l'approvisionnement en silex est très majoritairement local, avec les silex noirs et blonds du sénonien. Les cortex roulés de ces derniers attestent une collecte principalement dans les alluvions de la Vézère à seulement quelques centaines de mètres des abris, sous la forme de blocs entiers, débités 
en totalité dans les sites. Dans tous les cas ces silex représentent plus de $90 \%$ de l'ensemble des matériaux employés (de $90 \%$ dans le Gravettien final de l'abri Pataud à $96 \%$ dans l'Aurignacien des abris Blanchard et Castanet; Chiotti et al. 2013, 2015). Des matériaux d'origine plus lointaine sont toujours présents, mais en faibles proportions. Parmi eux, le silex campanien de la région de Bergerac (distant de 40 à $50 \mathrm{~km}$ des sites) est toujours le mieux représenté, surtout dans les sites du Gravettien final : il atteint un maximum de 7,7 \% à l'abri Pataud (Chiotti et al. 2013), alors qu'il ne dépasse pas 4,6\% dans l'Aurignacien (abri Blanchard, secteur 4/5). Ce silex est, par ailleurs, beaucoup mieux représenté dans les outillages que dans l'ensemble du matériel, avec un maximum de 32,3 à 36,8 \% dans l'outillage des niveaux de LaugerieHaute (Demars 1994). Ces matériaux sont représentés soit par des objets isolés, soit par de petits blocs préalablement préparés dont le débitage a été terminé sur les sites. D’autres matériaux lointains sont également présents dans la plupart des cinq sites : des silex turoniens de la région de Fumel (Haut-Agenais; c. $60 \mathrm{~km}$ ), des silex jaspoïdes de l'Hettangien du bassin de Brive (c. $45 \mathrm{~km}$ ) ou encore des silex cénozoïques du Périgord (10 à 30 km) (Demars 1994; Kong-Cho 1997; Pelegrin \& White 1998; Chiotti et al. 2013, 2015). Il faut également noter la présence rare de quelques matériaux encore plus lointains : du silex lacustre tertiaire de la bordure sud du Massif Central (100 à $120 \mathrm{~km}$ ) à Pataud et Castanet, du silex dit "Grains de Mil» de la région de Jonzac (CharenteMaritime; c. $140 \mathrm{~km}$ ) à Castanet et Blanchard, du silex maestrichtien à Lépidorbitö̈des du sud du Bassin Aquitain (90 km pour les alluvions de la Garonne à plus de $150 \mathrm{~km}$ pour les gîtes) à Laugerie-Haute (il s'agit du nucléus réutilisé en percussion cité précédemment) et un probable silex du turonien inférieur de Touraine $(c .180 \mathrm{~km})$ à Pataud (Chiotti et al. 2013, 2015, 2018). Ces matériaux sont représentés essentiellement par des objets isolés.

L'approvisionnement en MLHS destiné aux activités de percussion et en silex présente de fortes similitudes, avec dans les deux cas une très forte majorité de matériaux collectés à proximité immédiate des abris, dans les alluvions de la Vézère. Les matériaux exogènes sont mieux représentés parmi les silex que parmi les MLHS. Cela s'explique très certainement par le poids des matériaux à transporter. Les galets représentant un poids important sont très probablement collectés au plus près des abris. Il en va de même pour la plupart des silex sénoniens constituant la majorité de la production lithique, qui sont collectés sous la forme de blocs entiers. Les silex plus lointains au contraire sont transportés soit sous la forme de produits finis (outils ou supports bruts), soit sous la forme de blocs préalablement préparés, ce qui permet d'en réduire le poids, et donc le coût énergétique du transport.

Les quelques silex non locaux témoignent de distances d'approvisionnement beaucoup plus importantes que les MLHS percutés, pouvant ponctuellement dépasser les $150 \mathrm{~km}$. Cependant, il convient de nuancer cette constatation car si l'on regarde l'ensemble des MLHS présentes sur les sites, il apparait que certains MLHS destinés à d'autres usages que la percussion ont parfois des origines aussi lointaines (Chiotti et al. 2018).
COMPARAISONS GÉOGRAPHIQUES ET CHRONOLOGIQUES

Les différentes catégories d'objets percutés décrites ici ont des répartitions variables dans le temps et dans l'espace. Mais il semble que la plupart d'entre elles soient présentes sous des formes très similaires sur l'ensemble du Paléolithique supérieur (voire sur des périodes beaucoup plus longues) et sur de vastes territoires. Nous allons essayer de montrer, par quelques exemples, le caractère relativement universel de la plupart de ces objets.

Les percuteurs de taille sont sans aucun doute les plus répandus. Associés, de par leur fonction, à l'existence d'outils taillés, on les rencontre depuis l'apparition des premières industries humaines jusqu'au Néolithique, voire au-delà (voir par exemple Chavaillon 1979; Beaune 1993b, 2000), et sur l'ensemble de la planète.

Les percuteurs de concassage et les choppers utilisés comme percuteurs de concassage sont nombreux dans la littérature. Certains sont signalés dès le Paléolithique moyen, par exemple sur les sites de Neumark-Nord 2 en Allemagne (Pop et al. 2018), ou du Pech-de-l'Azé I (Carsac-Aillac, Dordogne; Soressi et al. 2008). Les récentes fouilles aurignaciennes de Chez-les-Rois (Mouthier-sur Boëme, Charente) ont livré quatre "percuteurs de fracturation", portant des traces de percussions sur des arêtes et sur des extrémités (Daulny 2006b) identiques à celles des pièces étudiés ici. Nous avons personnellement retrouvé des objets similaires dans le niveau Gravettien final de l'abri des Peyrugues (Orniac, Lot; Chiotti et al. 2018). P. Smith figure des chopper identiques dans les différents niveaux solutréens de Laugerie-Haute (Smith 1966). Le Magdalénien du Rocher de la Caille (St-Maurice-sur-Loire, Loire) a livré dix percuteurs de concassage parmi lesquels plusieurs portent des traces d'écrasement sur un tranchant aménagé de type chopper ou chopping-tool (Beaune \& Santallier 2003). Pour le Magdalénien, on peut également signaler le site de Gonnersdorf (Allemagne) qui livre des galets présentant des enlèvements unifaciaux ou bifaciaux accompagnés de fortes traces d'impacts (Bosinski 1979). De tels outils, portant de très nombres traces d'écrasement et de martèlement sur une de leurs arêtes ou de leurs extrémités sont également signalés dans l'Azilien de Pont-d'Ambon (Bourdeilles, Dordogne; Célérier \& Kervazo 1988; Beaune 1993a).

Les percuteurs de concassage et choppers utilisés en percussion sont donc présents au moins depuis le Paléolithique moyen et se retrouvent tout au long du Paléolithique supérieur. Ils présentent partout des traces très similaires qui semblent toujours correspondre à un même type d'usage (voir plus haut).

Les percuteurs minéraux tendres sont relativement rares dans la littérature, et ce malgré le fait que l'usage de ce type de percuteur soit désormais bien attesté dans les industries du Paléolithique supérieur par les stigmates identifiés sur le matériel taillé (Pelegrin 2000). On peut par exemple signaler une pièce similaire aux percuteurs en calcaire de Pataud et de Blanchard dans l'Ibéromaurusien d'Ifri n'Ammar (Maroc), qui est décrite comme un percuteur en chaille, de forme sphérique, légèrement irrégulière et facettée (Moser 2003). Bien que de nature différente (galets de forme non modifiée) deux percuteurs en calcaire tendre sont décrits dans l'Aurignacien de la grotte de Fumane (Italie; Caricola et al. 2018). 
Des pièces sphériques, généralement façonnées dans le calcaire local, ont été mises au jour dans des sites du Paléolithique moyen où elles apparaissent le plus souvent sous le terme de "bolas», mais aussi de «boules calcaires» ou encore de «sphéroïdes» (pour plus de détail, voir Chiotti et al. 2018). L'étude de l'industrie lithique du site de Chez Pinaud (Jonzac, Charente-Maritime) couplée à une approche expérimentale a permis de montrer que les pièces en question sont certainement des percuteurs (Roussel et al. 2009).

Les maillets sont présents tout au long du Paléolithique supérieur et sur l'ensemble du territoire européen. Ils sont particulièrement nombreux dans le site d'Isturitz où ils ont été décrits par S. de Beaune (1997). Ces objets souvent qualifiés de "compresseurs » sont en général peu nombreux, mais ils sont présents dans de très nombreux sites (voir Beaune 1997 pour un inventaire de ces objets).

Comme nous l'avons déjà signalé, les objets en silex taillés (en particulier des nucléus) réutilisés en percussion sont assez peu fréquents dans la littérature où ils sont simplement cités lors de la description des industries lithiques. Malgré cela, on en trouve des exemples durant tout le Paléolithique supérieur, voire même au-delà et dans toute l'Europe (Chiotti et al. 2018). On trouve par exemple de pièces très similaires à celles étudiées ici (en particulier aux pièces les plus percutées) dans le Gravettien et le Proto-solutréen de la grotte du Trilobite (Arcy-sur-Cure, Yonne; Bodu \& Renard 2013). Nous avons personnellement identifié un de ces objets très fortement percuté et complètement arrondi dans la collection du site de La Ferrassie (Savignac-de-Miremont, Dordogne) conservée au Musée de L'Homme, MNHN (données personnelles de l'auteur, non publié). D'autres sont signalées sur des sites russes : à Kostienki 4 - Aleksandrovskaya, contemporain du Gravettien (Rogatchev 1955) ou encore Kostienki 1 - Poliakov, contemporain de l'Aurignaco-Gravettien (Efimenko 1958). Dans ce dernier cas, l'auteur pense qu'il ne s'agit pas de nucléus, mais de pièces qui ont été taillées pour leur donner une forme appropriée. Bien qu'attestant généralement de percussions beaucoup moins intenses, des nucléus percutés sont présents sur de nombreux sites du Paléolithique ancien et moyen. À ces périodes, de telles traces de percussions sont aussi fréquemment présentes sur des bifaces (Claud et al. 2010; Thiébaut et al. 2010). Des objets très proches existent également en Amérique du Sud où ils sont décrits sous le terme de percuteurs sur arête. Il s'agit d' «outils sur nucléus de forme polyédrique massive dont une ou plusieurs arêtes ont été utilisées pour marteler, écraser, etc. " (Emperaire et al. 1963). Ces objets sont très fréquents dans les gisements postglaciaires de Patagonie et de Terre de Feu (Emperaire \& Laming 1961; voir Laming-Emperaire et al. 1972 pour des illustrations).

Quelle qu'en soit la finalité (voir plus haut), la réutilisation de nucléus en silex pour des activités de percussion, semble donc bien être, à la vue de ces quelques exemples, un phénomène très répandu, aussi bien dans le temps que dans l'espace.

Contrairement aux autres types d'objets décrits ici, les pièces intermédiaires en silex de grand module décrites à l'abri Castanet ne sont pas répandues. Nous n’avons pas connaissance de tels objets dans la littérature.
Les enclumes sont elles aussi des objets très répandus. On en connait depuis le début du Paléolithique jusqu’au Néolithique, et elles sont présentes dans le monde entier (Beaune 1993b). Elles ont pu servir de support à de nombreuses activités telles que le débitage du silex ou la fragmentation de différents matériaux (os, noix, bois, etc.; Beaune 2000).

\section{CONCLUSION}

Les cinq sites aurignaciens et gravettiens final de la vallée de la Vézère ont livré un total de 275 pièces lithiques portant des stigmates de percussions. À l'exception des pièces intermédiaires uniquement documentées à l'abri Castanet et des grattoirs carénés limités à l'Aurignacien, les différentes catégories d'outils identifiées sont présentes sur la plupart des sites étudiés, indépendamment de l'attribution de ces derniers à l'Aurignacien ou au Gravettien final.

En plus d'être abondant, le matériel de percussion découvert dans les sites étudiés est relativement diversifié d'un point de vue typologique. Les types d'outils et les stigmates qu'ils portent étant très similaires dans les deux cultures, il semble probable que pour un type d'outil donné les fonctions soient identiques.

Du point de vue des matériaux employés, les galets de quartz sont largement majoritaires quels que soient le site et la culture. On note néanmoins que le choix des matériaux peut fortement varier selon le type d'outil considéré. Ainsi, les choppers-percuteurs identifiés sont presque tous en quartz, tandis qu'à l'inverse, un grand nombre de matériaux - à l'exclusion notable du quartz - ont été utilisés comme maillets.

Dans la plupart des cas, les matériaux sélectionnés sont disponibles dans les alluvions de la Vézère, dont le cours est, pour tous les sites considérés, situé à proximité immédiate des abris. Ces alluvions constituent très probablement la source principale d'approvisionnement en MLHS. Cependant, certains matériaux tels que le trachyte n'y sont pas présents. Ceux-ci ont alors pu être collectés à l'échelle régionale, notamment dans la vallée de la Dordogne. Ces derniers matériaux représentant de quantités et des volumes limités, il est envisageable qu'ils aient simplement fait l'objet d'une collecte occasionnelle lors des déplacements des groupes.

Enfin, l'analyse des différentes séries étudiées pour les cinq sites a permis de montrer que l'outillage lié à la percussion est quantitativement beaucoup plus important que ne le laissait supposer les anciennes fouilles. Comme cela est désormais bien connu, les fouilles récentes ont montré que ce type de matériel avait été largement négligé lors des anciens travaux. De plus, les collectes effectuées dans les déblais des fouilles anciennement menées dans plusieurs de ces sites ont permis de compléter les anciennes collections et de montrer l'importance à la fois quantitative et informative de ces types d'outils.

\section{Remerciements}

Nous tenons ici à remercier tout particulièrement Randall White, Professeur à l'Université de New York, pour nous avoir offert l'occasion de participer aux fouilles et à l'étude du 
matériel lithique des sites de Castanet, Blanchard et Cellier, ainsi que toutes les personnes qui ont permis que ces fouilles se déroulent dans d'excellentes conditions, et notamment Romain Mensan, Sarah Ranlett, Amy Clark et Matthew Sisk. Un grand merci également à Jean-Jacques Cleyet-Merle, Directeur du Musée national de Préhistoire qui nous a permis d'accéder aux collections de D. Peyrony (Castanet et Laugerie-Haute Est) et de F. Bordes (Laugerie-Haute Est) qui sont conservées dans son institution. Nos remerciements vont également à Jean-Max Touron qui nous a autorisé à étudier la collection de l'abri Cellier.

L'opération archéologique programmée «L'occupation humaine de l'abri Pataud il y a 22000 ans " a été financée par le ministère de la Culture, DRAC Aquitaine, par le département de la Dordogne et par le Muséum national d'Histoire naturelle.

Les opérations archéologiques des abris Castanet, Blanchard et Cellier ont pu être menées à bien grâce aux soutiens de la United States National Science Foundation, la Direction régionale des affaires culturelles d'Aquitaine (DRAC-Aquitaine), le Partner University Fund, la L. S. B. Leakey Foundation, la Reed Foundation, la Rock Foundation, la Fine Foundation, l'UMI 3199-CNRS-NYU, l'Institute for Ice Age Studies, la Theodore Dubin Foundation, le département de la Dordogne, la New York University, la Fondation Fyssen et le programme Fulbright de l'état américain.

Nous tenons également à remercier les deux relecteurs anonymes pour leurs commentaires qui nous ont permis de parfaire ce manuscrit.

\section{RÉFÉRENCES}

BEAune S. DE 1993a. - L'abri sous roche de Pont d'Ambon à Bourdeilles (Dordogne) : IV, Le matériel lithique non taillé. Gallia Préhistoire 35: 112-137. https://doi.org/10.3406/galip.1993.2085

Beaune S. DE 1993b. - Nonflint Stone Tools of the Early Upper Paleolithic, in Knecht H., Pike-Tay A. \& White R. (eds), Before Lascaux: the complex record of the early Upper Paleolithic. CRC Press, Boca Raton: 163-191.

BEAUNE S. DE 1997. - Les galets utilisés au Paléolithique supérieur. Éditions du CNRS, Supplément à Gallia Préhistoire, XXXII, Paris, 298 p. https:/www.persee.fr/doc/galip_0072-0100_1997_sup_32_1

BEAUNE S. DE 2000. - Pour une archéologie du geste. Broyer, moudre, piler, des premiers chasseurs aux premiers agriculteurs. Éditions du CNRS, Paris, $231 \mathrm{p}$.

Beaune S. DE \& SANTALLIER D. 2003. — Les outils sur galet du site magdalénien du Rocher de la Caille, in Deloge H., Deloge L. \& BEAUNE S. A. DE (dirs), Le Rocher de la Caille: un site magdalénien de plein air au Saut-du-Perron Saint-Jean/Saint-Maurice-sur-Loire (Loire). Société préhistorique française, Mémoires de la Société préhistorique française, XXXI, Paris: 137-150. https://halshs. archives-ouvertes.fr/halshs-00722454/document

Bodu P. \& Renard C. 2013. — «L'ancien» Solutréen du Bassin parisien, quelques observations récentes, in BODU P., CHEHMANA L., Klaric L., Mevel L., Soriano S. \& Teyssandier N. (dirs), Le Paléolithique supérieur ancien de l'Europe du Nord-Ouest. Réflexions et synthèses à partir d'un projet collectif de recherche sur le centre et le sud du Bassin parisien, actes du colloque de Sens, 15-18 avril 2009. Société préhistorique française, Mémoires de la Société préhistorique française, LVI, Paris: 117-141.

BORDES F. 1958. - Nouvelles fouilles à Laugerie-Haute. Premiers résultats. L'Anthropologie 62 (3-4): 205-244.
Bordes F. 1978. — Le Protomagdalénien de Laugerie-Haute-Est (fouilles F. Bordes). Bulletin de la Société préhistorique française 75 (11-12): 501-521. https://doi.org/10.3406/bspf.1978.8537 BOSINSKI G. 1979. - Der Magdalenien Funfplatz Gönnersdorf 3. Die Behausungen von Gönnersdorf der Augrabung 1968. Franz Steiner Verlag GMBH, Wiesbaden, 220 p.

Bourrillon R., White R., Tartar É., Chiotti L., Mensan R., Clark A., Castel J.-C., Cretin C., Higham T., Morala A., Ranlett S., Sisk M. \& Comeskey D. J. 2018. — A new Aurignacian engraving from Abri Blanchard, France: Implications for understanding Aurignacian graphic expression in Western and Central Europe. Quaternary International 491: 46-64. https:// doi.org/10.1016/j.quaint.2016.09.063

Caricola I., Zupancich A., Moscone D., Mutri G., Falcucci A., Duches R., Peresani M. \& Cristiani E. 2018. — An integrated method for understanding the function of macro-lithic tools. Use wear, 3D and spatial analyses of an Early Upper Palaeolithic assemblage from North Eastern Italy. PLoS ONE 13: e0207773. https://doi.org/10.1371/journal.pone.0207773

CÉLÉRIER G. \& KeRVAZO B. 1988. - Livret-guide pour la visite du gisement de Pont d'Ambon, Bourdeilles. Excursion du colloque pour le Centenaire de la découverte de l'homme de Chancelade, Chancelade, 10-14 octobre 1988, 13 p.

Chavaillon J. 1979. - Essai pour une typologie du matériel de percussion. Bulletin de la Société préhistorique française 76 (8): 230-233. https://doi.org/10.3406/bspf.1979.5213

Chiotti L. \& Cretin C. 2011 . - Les mises en forme de grattoirs carénés / nucléus de l'Aurignacien de l'abri Castanet (Sergeac, Dordogne). Paleo 22: 69-84. https://doi.org/10.4000/ paleo. 2077

Chiotti L., Cretin C. \& Morala A. 2015. - Les industries lithiques des abris Blanchard et Castanet (Dordogne, France) : Données issues des fouilles 2005-2012, in WHITE R. \& BOURRILLON R. (dirs), avec la collaboration de BON F., Aurignacian Genius : art, technologie et société des premiers hommes modernes en Europe, actes du symposium international de New York, 8-10 avril 2013.P@lethnologie 7: 77-98. https://doi.org/10.4000/ palethnologie.741

Chiotti L., Morala A. \& Nespoulet R. 2018. - Usage domestique et symbolique du minéral au Gravettien final. Abri PataudLaugerie-Haute Est - Les Peyrugues. Musée national de Préhistoire, Paleo, numéro spécial, Les Eyzies-de-Tayac, 212 p.

Chiotti L., Nespoulet R., Henry-Gambier D., Morala A., Vercoutère C., Agsous S., Lenoble A., Marquer L. \& Grimaud-Hervé D. 2009. - Statut des objets «extra-ordinaires» du Gravettien final de labri Pataud (Les Eyzies, Dordogne) : objets abandonnés dans l'habitat ou dépôt intentionnel ?, in Bonnardin S., Hamon C., LauWers M. \& Quilliec B. (eds), Du matériel au spirituel. Réalités archéologiques et historiques des "dépôts" de la Préhistoire à nos jours, XXIXe Rencontres internationales d'archéologie et d'histoire d'Antibes, Juan-les-Pins, 16-18 octobre 2008. Éditions APDCA, Antibes: 29-46.

Chiotti L., Nespoulet R., Henry-Gambier D., Vercoutère C., Crépin L., Lebon M., BeCK L., MÜller K. \& Reiche I. 2014. Un comportement funéraire original au Gravettien final. Bilan des analyses et études 2005-2011 de la couche 2 de l'abri Pataud (Les Eyzies-de-Tayac, Dordogne), in PAILlet P. (dir.), Les arts de la Préhistoire: micro-analyses, mises en contexte et conservation, actes du colloque "Micro-analyses et datations de l'art préhistorique dans son contexte archéologique", MADAPCA, Paris, 16-18 novembre 2011. Musée national de Préhistoire, Paleo, numéro spécial, Les Eyzies-de-Tayac: 183-193.

Chiotti L., Nespoulet R., Morala A. \& Guillermin P. 2013. - Données typo-technologiques de l'industrie lithique, in Nespoulet R., Chiotti L. \& Henry-Gambier D. (dirs), Le Gravettien final de l'abri Pataud (Dordogne, France). Fouilles et études 2005-2009. Archaeopress, BAR International Series, no. 2458, Oxford: 111-126. 
Claud É., Mourre V., Thiébaut C. \& Brenet M. 2010. Le recyclage au Paléolithique moyen. Des bifaces et des nucléus utilisés comme percuteurs, in Recyclage et remploi. Archéopages 29: 6-15. http://dolia.inrap.fr/flora/ark:/64298/0139940

Collie L. G. 1928. - Department of the Dordogne, France, in Collie L. G. (ed.), The Aurignacians and their Culture. The Logan Museum, Logan Museum Bulletin, vol. I., no. 1, Beloit College, Beloit: 62-71.

DAULNY L. 2006a. - Les vestiges sur galets, bloc et plaquettes, témoins des comportements des modes de vie des hommes préhistoriques, in DACHARY M. (dir.), Les Magdaléniens à Duruthy. Qui étaient-ils? Comment vivaient-ils? Catalogue de l'exposition présentée à l'abbaye d'Arthous à Hastingues, 7 octobre-10 décembre 2006. Centre Départemental du Patrimoine, Hastingues: 52-59.

DAULNY L. 2006b. - L'apport de l'analyse des témoins minéraux sélectionnés pour la reconstitution des comportements et des modes de vie des hommes de l'Aurignacien des Rois (Mouthierssur-Boëme, Charente). Les vestiges lithiques hors silex des fouilles "2005-2006», couches «1», « 2 » et " 3 », in D'ERrICO F. \& VAnHAeren M. (dirs), Rapport de fouille aux Rois. Rapport remis au Service Régional de l'Archéologie de Poitou-Charente.

DAUlNY L. \& DACHARY M. 2009. - Approche comportementale du Magdalénien d'après l'étude techno-fonctionnelle de l'outillage lithique hors silex; la grotte de Bourrouilla (Arancou, Pyrénées-Atlantiques, France), in STERnKe F., Eigeland L. \& COSTA L.-J. (eds), L’utilisation préhistorique de matières premières lithiques alternatives. Anciens préjugés, nouvelles perspectives, actes du XVe Congrès de l'Union internationale des Sciences préhistoriques et protohistoriques, Lisbonne, 4-9 septembre 2006. Archaeopress, BAR International Series, no. 1939, Oxford: 21-28.

DelluC B. \& DelluC G. 1981. - La dispersion des objets de l'abri Blanchard (Sergeac, Dordogne). Bulletin de la Société de Recherches et d'Études préhistoriques des Eyzies 30: 77-95.

Demars P.-Y. 1994. - L'économie du silex au Paléolithique supérieur dans le nord de l'Aquitaine. Analyse, synthèse et interprétations. Thèse de doctorat d'État, Université Bordeaux 1, Bordeaux, 2 vol., 270 et 549 p.

Didon L. 1911. - L'abri Blanchard des Roches (commune de Sergeac). Gisement aurignacien moyen. Bulletin de la Société Historique et Archéologique du Périgord 38: 246-261 et 321-345.

EFIMENKO P. P. 1958. - Kostienki I. Académie des Sciences d'URSS, Moscou-Léningrad, 483 p. (en russe).

EMPERAIRE J. \& LAMING A. 1961. — Les gisements des îles Englefield et Vivian dans la mer d'Otway (Patagonie australe). Journal de la Société des américanistes 50: 7-75. https://doi.org/10.3406/ jsa.1961.2477

Emperaire J., Laming-Emperaire A., Reichlen H. \& PoulainJosien T. 1963. - La grotte Fell et autres sites de la région volcanique de la Patagonie chilienne. Journal de la Société des américanistes 52: 167-254. https://doi.org/10.3406/jsa.1963.1996

Henri-MarTin L. 1910. - Recherches sur l'évolution du Moustérien dans le gisement de La Quina (Charente). Vol.1: Industrie osseuse. Schleicher Frères, Paris, 315 p. https://gallica.bnf.fr/ark:/12148/ bpt6k9615893h?rk=21459;2

KonG-Cho S. 1997. — Étude de l'industrie lithique du niveau 2 dite Protomagdalénienne de l'abri Pataud (Les Eyzies, Dordogne): étude technologique et typologique. Thèse de doctorat, Muséum national d'Histoire naturelle, Paris, $374 \mathrm{p}$.

Laming-Emperaire A., LaVallée D. \& Humbert R. 1972. - Le site de Marazzi en Terre de Feu. Objets et Mondes XII (2): 225-244.

LE BRUN-Ricalens F. 2006. - Les pièces esquillées : état des connaissances après un siècle de reconnaissance. Paleo 18: 95-114. https://doi.org/10.4000/paleo.181

Leroi-Gourhan A. 1997. - Dictionnaire de la Préhistoire. 2e édition. Quadrige / Presses Universitaires de France, Paris, 1277 p.

MOSER J. 2003. - La Grotte d'Ifri n'Ammar. Tome 1: L'Ibéromaurusien. Linden Soft, Deutschen Archäologischen Instituts, coll. Forschungen zur allgemeinen und vergleichenden Archäologie, no. 8, Köln, 163 p.
Mourre V. \& JARry M. (dirs) 2009-2010. — «Entre le marteau et l'enclume..." La percussion directe au percuteur dur et la diversité de ses modalités d'application. Actes de la table ronde de Toulouse, 15-17 mars 2004. SAMRA, Les Eyzies-de-Tayac. Paleo, numéro spécial, $237 \mathrm{p}$.

Movius H. L. JR. 1975 (ed.). - Excavation of the abri Pataud, Les Eyzies (Dordogne): Contributors. Peabody Museum, coll. American School of Prehistoric Research Bulletins, no. 30, Harvard University, Cambridge, 305 p.

Movius H. L. JR. 1977. - Excavation of the abri Pataud, Les Eyzies (Dordogne): Stratigraphy. Peabody Museum, Harvard University, coll. American School of Prehistoric Research, no. 31, Cambridge, $167 \mathrm{p}$.

Nespoulet R., Chiotti L. \& Henry-Gambier D. (dirs) 2013. Le Gravettien final de l'abri Pataud (Dordogne, France). Fouilles et études 2005-2009. Archaeopress, BAR International Series, no. 2458 , Oxford, 217 p.

Pelegrin J. 2000. - Les techniques de débitage laminaire au Tardiglaciaire : critères de diagnose et quelques réflexions, in L'Europe centrale et septentrionale au Tardiglaciaire, actes de la table ronde de Nemours, 13-16 mai 1997. Musée de Préhistoire d'Île de France, Mémoires du Musée de Préhistoire d'Île de France, no. 7, Nemours: 73-86.

Pelegrin J. \& White R. 1998. — Abri Castanet (Sergeac, Dordogne). Rapport de fouille programmée triannuelle (1996-1998). Rapport remis au SRA Aquitaine, 57 p. + annexes.

Peyrony D. 1935. - Le gisement Castanet, Vallon de Castelmerle, commune de Sergeac (Dordogne), Aurignacien I et II. Bulletin de la Société préhistorique française 32 (9): 418-443. https://doi. org/10.3406/bspf.1935.6122

PEYRONY D. 1946. - Périgord. Le gisement préhistorique de l'abri Cellier, au Ruth, commune de Tursac (Dordogne). Gallia 4: 294-301. https://www.persee.fr/doc/galia_0016-4119_1946_ num_4_1_2005

Peyrony D. \& Peyrony E. 1938. - Laugerie-Haute près des Eyzies (Dordogne). Masson, Archives de l'Institut de paléontologie humaine, no. 19, Paris, 84 p.

Pop E., Charalampopoulos D., Arps C. S., Verbaas A., RoeBROEKS W., Gaudzinski-Windheuser S. \& Langejans G. 2018. - Middle Palaeolithic Percussive Tools from the Last Interglacial Site Neumark-Nord 2/2 (Germany) and the Visibility of Such Tools in the Archaeological Record. Journal of Palaeolithic Archaeology 1 (2): 81-106. https://doi.org/10.1007/ s41982-018-0008-8

Rogatchev A. N. 1955. - Kostienki 4. L'habitat d'Aleksandrovskaya de l'âge de la pierre ancienne à Kostienki sur le Don. Académie des sciences de l'URSS, coll. Matériaux et recherches sur l'Archéologie de l'URSS, no. 45, Moscou-Leningrad, 164 p. (en russe).

Roussel M., Bourguignon L. \& Soressi M. 2009. - Des blocs de calcaire utilisés comme percuteurs dès le Moustérien? L'exemple de Jonzac (Charente-Maritime), in Dumas C., Roussel B. \& TEXIER P.-J. (dirs), Langage de pierre. La restitution du geste en archéologie préhistorique, actes du colloque européen des Baux de Provence, 10 octobre 2009. Éditions Maison Cazenave, Musée des Baux de Provence, Les Baux de Provence: 11-13.

SALOMON H. 2009. - Les matières colorantes au début du Paléolithique supérieur : sources, transformation et fonctions. Thèse de doctorat, Université Bordeaux 1, Bordeaux, 432 p. https:// hal.archives-ouvertes.fr/tel-02430482/document

Sestier C. \& BonTEMPS C. 2003. — Les bouchardes en matériaux tenaces : observations archéologiques et expérimentales, in Les matières premières lithiques en préhistoire, actes de la table ronde internationale d'Aurillac, 20-22 juin 2002. Préhistoire du SudOuest, supplément 5: 307-315.

Sмiтh P. E. L. 1966. - Le Solutréen en France. Imprimerie Delmas, Publications de l'Institut de Préhistoire de l'Université de Bordeaux, mémoire no. 5, Bordeaux, 449 p. 
SonneVILle-Bordes D. DE 1960. — Le Paléolithique supérieur en Périgord. Imprimerie Delmas, Bordeaux: 2 vol., 274 et 558 p.

Soressi M., Rendu W., Texier J.-P., Claud É., Daulny L., D’Errico F., Laroulandie V., Maureille B., Niclot M., Schwortz S. \& Tillier A.-M. 2008. — Pech-de-l'Azé I (Dordogne, France) : nouveau regard sur un gisement moustérien de tradition acheuléenne connu depuis le XIXe siècle, in JAUBERT J., BORDES J.-G. \& ORTEGA I. (dirs), Les sociétés paléolithiques dans un grand Sud-ouest : nouveaux gisements, nouveaux résultats, nouvelles méthodes, actes des journées de la Société préhistorique française, Talence, 24 et 25 novembre 2006. Société préhistorique française, Mémoires de la Société préhistorique française, XLVII, Paris: 95-131.

Tartar É., White R., Chiotti L., Cretin C. \& Mensan R. 2014. - Quel(s) Aurignacien(s) à l'abri Blanchard (Sergeac, Dordogne)? Données des collections d'industrie osseuse conservées aux États-Unis et retour sur le terrain. Paleo 25: 309-331. https://doi.org/10.4000/paleo.2836

Thiébaut C., Claud É., Mourre V., Chacon M.-G., Asselin G., Brenet M. \& Paravel B. 2010. - Le recyclage et la réutilisation de nucléus et de bifaces au Paléolithique moyen en Europe occidentale : quelles fonctions et quelles implications culturelles? P@lethnologie 2010:3-41. https://doi.org/10.4000/ palethnologie. 588

TiXIer J., InIZAN M.-L. \& Roche H. 1980. — Préhistoire de la pierre taillée 1 terminologie et technologie. Cercle de recherches et d'études préhistoriques, Antibes, $120 \mathrm{p}$.

Vialou D. 2004 (dir.). - La Préhistoire. Histoire et dictionnaire. Robert Laffont, coll. Bouquins, Paris, 1637 p.

White R. 2006 (dir.). - Abri Castanet, Secteur Sud (Commune de Sergeac, Dordogne). Rapport de fouille programmée. Année 2006. Rapport remis au SRA Aquitaine, 184 p.
White R. 2010 (dir.). - Abri Castanet, Secteur Sud et d'autres interventions dans le vallon de Castel-Merle (Commune de Sergeac, Dordogne). Rapport de fouille programmée. Année 2010. Rapport remis au SRA Aquitaine, $280 \mathrm{p}$.

WHITE R. 2011 (dir.). - Abri Blanchard, opération de diagnostic et autres interventions dans le vallon de Castel-Merle (commune de Sergeac, Dordogne). Rapport de fouilles programmées. Année 2011. Rapport remis au SRA Aquitaine, 189 p.

White R. 2012 (dir.). - Abri Blanchard et Abri Castanet secteur central. Vallon de Castel-Merle (commune de Sergeac, Dordogne). Rapport de fouilles programmées et opération de diagnostic. Année 2012. Rapport remis au SRA Aquitaine, 143 p.

White R. 2014 (dir.). - Abri Cellier (Commune de Tursac, Dordogne). Année 2014. Rapport d'opération de diagnostic. Rapport remis au SRA Aquitaine, $201 \mathrm{p}$.

White R., Bourrillon R., Mensan R., Clark A., Chiotti L., Higham T., Ranlett S., Tartar É., Morala A. \& Soulier M.-C. 2018. - Newly discovered Aurignacian engraved blocks from Abri Cellier: History, context and dating. Quaternary International 498: 99-125. https://doi.org/10.1016/j.quaint.2017.02.001

White R., Mensan R., Bourrillon R., Cretin C., Higham T., Clark A., Sisk M., Tartar É., Gardère P., Goldberg P., Pelegrin J., Valladas H., Tisnerat-Laborde N., Sanoit J. De, Chambellan D. \& Chiotti L. 2012. - Context and dating of Aurignacian vulvar representations from Abri Castanet, France. Proceedings of the National Academy of Sciences of the United States of America 109 (22): 8450-8455. https://doi.org/10.1073/ pnas.1119663109

Woods A. D. 2011. - The effects of lithic raw material quality on Aurignacian blade production at Abri Cellier. Doctoral dissertation, University of Iowa, Iowa City, 182 p. https://ir.uiowa. edu/etd/1111/ 